\title{
Decimalization, Realized Volatility, and Market Microstructure Noise
}

\author{
Tommi A. Vuorenmaa \\ University of Helsinki and HECER
}

Discussion Paper No. 217

May 2008

ISSN 1795-0562

HECER - Helsinki Center of Economic Research, P.O. Box 17 (Arkadiankatu 7), FI-00014 University of Helsinki, FINLAND, Tel +358-9-191-28780, Fax +358-9-191-28781,

E-mail info-hecer@helsinki.fi, Internet www.hecer.fi 


\title{
Decimalization, Realized Volatility, and Market Microstructure Noise*
}

\begin{abstract}
This paper studies empirically the effect of decimalization on volatility and market microstructure noise. We apply several non-parametric estimators in order to accurately measure volatility and market microstructure noise variance before and after the final stage of decimalization which, on the NYSE, took place in January, 2001. We find that decimalization decreased observed volatility by decreasing noise variance and, consequently, increased the significance of the true signal especially in the trade price data for the high-activity stocks. In general, however, most of the found increase in the signal-to-noise ratio is explainable by confounding and random effects. We also find that although allowing for dependent noise can matter pointwisely, it does not appear to be critical in our case where the estimates are averaged over time and across stocks. For that same reason rare random jumps are not critical either. It is more important to choose a proper data type and prefilter the data carefully.
\end{abstract}

JEL Classification: C14, C19.

Keywords: Decimalization; Market microstructure noise; Realized volatility; Realized variance; Tick size; Ultra-high-frequency data.

Tommi A. Vuorenmaa

Department of Economics

University of Helsinki

P.O. Box 17 (Arkadiankatu 7)

FI-00014 University of Helsinki

FINLAND

e-mail: tommi.vuorenmaa@helsinki.fi

\footnotetext{
${ }^{*}$ Research carried out while visiting the Bendheim Center for Finance at Princeton University in 2006-07. I am grateful to Yacine Aït-Sahalia for the chance to work in this exciting environment and for his valuable comments during the visit. I am also grateful to the participants of the Bendheim Center for Finance Student Research Workshop and Pentti Saikkonen (University of Helsinki) for further comments. Finally, I thank Roger Laeven (Tilburg University) for many interesting discussions. All remaining errors are my responsibility. Financial support from OPryhmän Tutkimussäätiö, Research Unit of Economic Structures and Growth (RUESG), Suomen Arvopaperimarkkinoiden Edistämissäätiö, and Thanks to Scandinavia Foundation are gratefully acknowledged.
} 


\section{Introduction}

The New York Stock Exchange (NYSE) completed its long anticipated change from fractional pricing to decimal pricing on January 29, 2001. This process is known as "decimalization." It is accompanied by a reduction in the minimum price variation called the tick size which in this case is from a sixteenth of a dollar to one cent. A reduction in tick size can have significant effects on other variables because it removes constraints on pricing and makes the cost of obtaining price priority smaller. This in turn may change the strategic behavior of the players in the market. The effect of decimalization on the quoted bid-ask spread, trading volume, and alike are well documented. The effects on more elaborate concepts are not as clear, however. Although volatility is almost unanimously reported to decrease [see, e.g., Ronen and Weaver (2001)], most of the empirical studies use unprecise estimation methods and sparsely sampled data that weaken the results. And with only very few exceptions [He and $\mathrm{Wu}(2005)]$ do these studies decompose volatility in any way.

In theory, price discreteness forces the observed price to deviate from the "true" price [see, e.g., Gottlieb and Kalay (1985), Cho and Frees (1988), and Easley and O'Hara (1992)]. As a consequence, the observed volatility is upward biased relatively to the true volatility by amount that depends on the tick size [see Harris (1990a)] and the sampling frequency [see, e.g., Gottlieb and Kalay (1985)]. Because decimalization alleviates rounding errors, the difference between the true and the observed price should narrow. This should damp the observed volatility but leave the true volatility intact. Hansen and Lunde (2006) find some evidence in this direction.

In this paper, we let the observed volatility to consist of two additive components: the true volatility and the market microstructure noise variance. This decomposition allows us to estimate them separately. If noise would not be separated out, the estimated volatility would depend on the sampling frequency through the noise term and cause trouble in subsequent modeling. We now want to find out if decimalization affects the true volatility and noise and their relative strength significantly. In order to accomplish 
this, we use elaborate econometric estimation methods that allow us to extract useful information from ultra-high-frequency (UHF) data. We take advantage of the fact that the NYSE decimalization was carried out in a manner that resembles a controlled natural experiment. We also use mixed-effect panel regressions to ensure that the found effects are not caused by confounding factors or randomness.

A key point is to first estimate the true volatility accurately. As noted for example by Cho and Frees (1988), the sample standard deviation underestimates the true standard deviation even in the absence of noise. We use several non-parametric estimators that have desirable statistical properties and are yet flexible and simple to calculate. In particular, if the market microstructure noise is IID, then the two-scale realized volatility (TSRV) estimator [Zhang, Mykland, and Aït-Sahalia (2005)] is consistent and unbiased. Similarly, the re-scaled realized volatility estimator provides consistent noise variance estimates. Both variance measures carry economically significant information [Bandi and Russell (2006)]. Their ratio, "signal-to-noise," allows us to evaluate changes in the composition of volatility.

We also let the market microstructure noise to be serially correlated. The generalized TSRV [Aït-Sahalia, Mykland, and Zhang (2006)] and the multi-scale realized volatility [Zhang (2005)] estimators are then still consistent and unbiased. The latter estimator, in particular, is able to account for time varying noise properties. Such flexibility is welcome because the properties of noise have been shown to vary over time and data type [see Hansen and Lunde (2006)]. We demonstrate how the volatility estimators cope with the change in tick size. This also enables us to study if decimalization affects the trade price and midquote estimates differently.

Finally, because jumps can have a deteriorating effect on the estimates of volatility and market microstructure noise variance [see Fan and Wang (2006)], we investigate the presence of jumps by a simple and direct test recently proposed by Aït-Sahalia and Jacod (2006). We find that jumps do exist, as expected, and that their impact may be different in event and calendar time. We then remove the jumps using different thresholds. Because true jumps (triggered by news, for example) are relatively rare 
random events, and because in our analysis we take averages over time and across stocks, we do not expect to see qualitatively significant changes in our results.

The empirical results of this paper show how a tick size reduction affects volatility and market microstructure noise. As argued by Harris (1990a), price discreteness can produce significant biases especially when small variance components are being identified from UHF data. This is relevant for example to risk management and options pricing [see Figlewski (1997)]. Our results should also be of interest to stock exchanges and institutions that work on to improve the efficiency of the markets. International volatility spillovers combined with the recent collusions between major stock exchanges highlight the need for rules that help to weigh down excess volatility (consider the NYSE-Euronext and Nasdaq-OMX mergers). In the future it may for example be that a tick size different from one cent is found optimal or that more advanced trading mechanisms are introduced in order to reduce market microstructure noise. After all, stock prices should ideally reflect all known information and not noise [see Black (1986) and Amihud and Mendelson (1987)].

The structure of this paper is as follows. In Section 1, we review the related decimalization literature. In Section 2, we describe the estimators we use in the empirical study. The data are described in Section 3. In Section 4, we report the empirical results. We conclude in Section 5. Appendix includes additional tables with volatility estimates and also illustrates the idea of subsampling and averaging that the estimators are based on.

\section{Review of decimalization effects}

This section gives a brief review of the relevant decimalization literature. [A more general survey can be found for example in Harris (1997, 1999) and NYSE (2001).] Notice that different methods and data sets make comparisons of many decimalization studies rather tricky. Market structure alone can have a significant effect on market characteristics [see Huang and Stoll (2001)]. Furthermore, a tick size decrease from 
eights to sixteenths does not necessarily have the same effect as a tick size decrease from sixteenths to cents.

The most direct evidence concerns the (bid-ask) spread. Both absolute and relative spreads have been found to decrease. As predicted for example by the Harris (1994) model, Ahn, Cao, and Choe (1996) and Ronen and Weaver (2001) find that the tick size decrease from eights to sixteenths reduced the quoted and effective spreads on the American Stock Exchange (AMEX). Similar evidence is reported for the change to five cents on the Toronto Stock Exchange (TSE) [e.g., Bacidore (1997)], from eights to sixteenths on the NYSE [Ricker (1998), Bollen and Whaley (1998), and Goldstein and Kavajecz (2000)], and from sixteenths to cents on the NYSE and the Nasdaq [Bessembinder (2003)]. Goldstein and Kavajecz (2000) and Bessembinder (2003) note that the spread decrease is largest for the most active stocks.

The Harris (1994) model also predicts a decrease in quoted depth and an increase in trading volume. Ahn, Cao, and Choe (1996) and Ronen and Weaver (2001) however find no significant changes in quoted depth or trading activity on the AMEX. On the other hand, Bacidore (1997) and Porter and Weaver (1997) find depth to decrease but trading volume to remain constant on the TSE. Ricker (1998), Bollen and Whaley (1998), Goldstein and Kavajecz (2000), and Bacidore, Battalio, and Jennings (2001) report decreases in quoted depth on the NYSE. van Ness, van Ness, and Pruitt (1999) report increases in the number of trades and volume. Chakravarty, van Ness, and van Ness (2005) report an increase in the volume of small sized trades.

Ricker (1998), Bollen and Whaley (1998), and Bacidore, Battalio, and Jennings (2001) find that decimalization improved liquidity on the NYSE. Bessembinder (2003) does not find evidence of liquidity problems on the Nasdaq either. It is indisputable, however, that displayed liquidity is decreased because of more order cancellations and smaller limit order sizes. NYSE (2001), among others, concludes that market transparency diminished. Jones and Lipson (2001) point out that the spread is no longer a sufficient statistic of market quality for large investors. They report increased average execution costs for a group of institutional investors on the NYSE due to the change 
from eights to sixteenths. Similar evidence is presented by Goldstein and Kavajecz (2000). In contrast, Chakravarty, Panchapagesan, and Wood (2005) do not find evidence of liquidity problems due to change to cents using NYSE Plexus data [in line with Bacidore, Battalio, and Jennings (2003)]. They suggest that large investors may have started using more cautious execution strategies.

Because liquidity does not appear to be adversely affected (at least not too much), decimalization is unlikely to increase volatility. Notice, however, that the great recent increase of algorithmic trading [see Economist (2007b)] - partly fueled by the diminished market transparency and the higher market making costs due to decimalization itself may have actually made the markets more sensitive to bad news. Trading algorithms can trigger sell-offs which in turn may cause losses of liquidity as happened for example in October 1987 when the widely adopted "portfolio insurance" strategies fully kicked in. More recent evidence is from February 2007 when automated trading led to severe order-routing problems on the NYSE [see Economist (2007a)]. These instances are of course expected to be rare by standard measures. ${ }^{1}$ In "normal periods" decimalization is likely to decrease volatility due to the smaller impact of price discreteness. ${ }^{2}$ Indeed, Ronen and Weaver (2001) and Bessembinder (2003) report volatility reductions on the AMEX, the NYSE, and the Nasdaq. Both studies proxy volatility by standard deviation or variance of midquote returns (the former also uses daily closing midquotes). ${ }^{3}$ They do not find exogenous market trends to be responsible for the decrease. Chakravarty, Wood, and van Ness (2004) report similar findings for portfolio volatility constructed from one minute intraday returns that are volume weighted.

It remains to identify what factors actually reduced volatility. A volatility reduction may be due to many different components such as the bid-ask bounce, price adjust-

\footnotetext{
${ }^{1}$ There is also evidence that algorithmic trading improves liquidity instead of decreasing it [see Hendershott, Jones, and Menkveld (2008)], that is, at least during "troubless" normal times.

${ }^{2}$ Ikenberry and Weston (2003) and Chung, van Ness, and van Ness (2004) however find significant clustering to five and ten cents after decimalization so that the impact may not be full. Clustering is not that surprising, though, because it simplifies price negotiation, among other things [see, e.g., Harris (1991)].

${ }^{3}$ As noted for example by Cho and Frees (1988), Jensen's inequality can be used to show that the sample standard deviation is a downward biased estimator of the true standard deviation. Gottlieb and Kalay (1985) find that the portfolio volatility estimates are nearly unbiased, however.
} 
ments to large trades, price discreteness, and so on. Market microstructure noise can be driven by information asymmetries between the traders and the market maker. Not surprisingly, identification of the factors is hard in practice. Conflicting evidence of for example whether adverse selection was increased or decreased exists [see Zhao and Chung (2006), Chakravarty, van Ness, and van Ness (2005), and Bacidore (1997)]. Perhaps most relevantly to us, $\mathrm{He}$ and $\mathrm{Wu}$ (2005) decompose the variance of price changes into public news, price discreteness, and bid-ask spreads using the method of Madhavan, Richardson, and Roomans (1997). They find a significant variance decline due to price discreteness and spreads. Gibson, Singh, and Yerramilli (2003) furthermore find that the spread reduction on the NYSE is due to a decrease in the order-processing component (and that inventory and adverse selection components remain significant). Engle and Sun (2005) find that in the decimalized NYSE as much as $86 \%$ of the variance of market microstructure noise in transactions can be due to variation in the informational component (the rest due to the non-informational component). In this paper, however, we treat market microstructure noise as a one unit and do not attempt to separate out its components.

\section{Estimators}

The framework in which we operate is standard. It can be viewed as a reduced form of structural market microstructure models such as the model of Madhavan, Richardson, and Roomans (1997) [see the discussion in Hasbrouck (1996)]. We now shortly review it.

Let the observed price, $Y$, consist of the latent true price $X$ and noise $\epsilon$ :

$$
Y_{t_{i}}=X_{t_{i}}+\epsilon_{t_{i}}
$$

The noise term is commonly assumed to be IID with mean zero and finite variance.

It includes transient factors such as the bid-ask bounce, price discreteness, inventory 
effects, and so on. In the IID case the first-order autocovariance of the (observed) returns can be shown to be $-\mathbb{E} \epsilon^{2}$ (and zero afterwards) [see, e.g., Aït-Sahalia, Mykland, and Zhang (2006)]. Because trades and quotes tend to cluster over time, this framework has been extended to include serially dependent noise. Hansen and Lunde (2006) argue it to be particularly relevant with very frequent sampling in a decimalized market like ours. We take dependent noise into account by using proper estimation methods (described below).

In Eq. (1), the true (generally unobserved) (log)price $X$ is standardly assumed to follow an Itô process,

$$
d X_{t}=\mu_{t} d t+\sigma_{t} d B_{t}
$$

with $\sigma_{t}$ possibly stochastic and $B_{t}$ the standard Brownian motion. ${ }^{4}$ Changes in the true price are believed to be information driven and they are permanent. This is in line with the view that the true price process should be positively correlated [see, e.g., Amihud and Mendelson (1987)]. Standardly, the true price is also assumed to be independent of the noise process. Although Hansen and Lunde (2006) argue that in practice negative dependence exists especially in the midquote data due to severe asymmetric information effects [see, e.g., Glosten and Milgrom (1985) and Madhavan, Richardson, and Roomans (1997)], in this paper we maintain the assumption of independence [for more discussion, see Aït-Sahalia, Mykland, and Zhang (2005b)].

If volatility is stochastic, it is important in many financial applications (e.g., in option pricing) to estimate the so-called integrated volatility (IV),

$$
\langle X, X\rangle_{T}=\int_{0}^{T} \sigma_{t}^{2} d t
$$

as accurately as possible. Without any noise arising from market microstructure, real-

\footnotetext{
${ }^{4}$ Itô processes include for example the Ornstein-Uhlenbeck process as a special case. The true price process could also be a more general semimartingale and still have its quadratic variation well defined [see, e.g., Protter (2005)]. A semimartingale with jumps however warrants special attention.
} 
ized volatility (RV), also known as quadratic variation (in the theoretical limit),

$$
[Y, Y]_{T}^{(a l l)}=\sum_{i=0}^{n-1}\left(Y_{t_{i+1}}-Y_{t_{i}}\right)^{2}
$$

over the observed prices at times $0=t_{0}<t_{1}<\cdots<t_{n}=T$, provides a precise estimate of IV as $\Delta t \rightarrow 0$ (sampling higher). ${ }^{5}$ In the presence of noise it does not, however. With IID noise, RV provides a consistent estimate of the noise variance instead [see Zhang, Mykland, Aït-Sahalia (2005) and Bandi and Russell (2003)]:

$$
\frac{1}{2 n}[Y, Y]_{T}^{(a l l)}=\widehat{\mathbb{E} \epsilon^{2}}
$$

We call this noise variance estimator the re-scaled realized volatility (RSRV). Because of its upward biasedness [see Oomen (2005b) and Hansen and Lunde (2006)], we also present a popular unbiased noise variance estimator later on (based on the aforementioned fact that the first-lag autocovariance term of returns equals $\left.-\mathbb{E} \epsilon^{2}\right) .{ }^{6}$

Several more precise estimators for IV have been proposed [see, e.g., Zhou (1996)]. Although most of them provide an unbiased estimate of IV, only few of them are consistent. In this paper we use three estimators that are not only unbiased but also consistent. These estimators all are based on the idea of subsampling and averaging, and although they start to be well known by now, we next describe them in some detail. The reader may find the details useful in the empirical section where we carry out some qualitative robustness checks.

The TSRV estimator of Zhang, Mykland, and Aït-Sahalia (2005) is defined as

$$
\widehat{\langle X, X}_{T}^{(t s r v)}=[Y, Y]_{T}^{(K)}-\frac{\bar{n}}{n}[Y, Y]_{T}^{(a l l)},
$$

\footnotetext{
${ }^{5}$ Typically, "realized volatility" and "realized variance" refer to the same quantity. Keep in mind, however, that in finance literature volatility often refers to standard deviation (of returns) rather than to variance. We nevertheless prefer to use the term realized volatility here.

${ }^{6}$ If negative dependence between the true price and noise truly exists, as suggested by Hansen and Lunde (2006), it should reduce the upward biasedness of the RSRV estimator for active stocks.
} 
where

$$
[Y, Y]_{T}^{(K)}=\frac{1}{K} \sum_{i=0}^{n-K}\left(Y_{t_{i+K}}-Y_{t_{i}}\right)^{2},
$$

$\bar{n}=(n-K+1) / K$, and $1<K \leq n$. If the noise is IID and $K$ is suitably chosen relatively to $n$, then the TSRV estimator is consistent, asymptotically unbiased, and normal [see Zhang, Mykland, and Aït-Sahalia (2005)]. [For the reader new to the idea of subsampling and averaging, we illustrate the calculation of the first sum in Eq. (3) by two numerically equal ways in Appendix A.1.]

The generalized TSRV (GTSRV) estimator proposed by Aït-Sahalia, Mykland, and Zhang (2006) allows for serially dependent noise. It is defined as

$$
\widehat{\langle X, X}_{T}^{(g t s r v)}=[Y, Y]_{T}^{(K)}-\frac{\bar{n}_{K}}{\bar{n}_{J}}[Y, Y]_{T}^{(J)}
$$

$\bar{n}_{K}=(n-K+1) / K$, similarly for $\bar{n}_{J}$, and $1 \leq J<K \leq n$. The GTSRV estimator reduces the impact of dependent noise by slowing down the "fast time-scale." It is consistent for suitable choices of $J$ and $K$ [see Aït-Sahalia, Mykland, and Zhang (2006)]. Setting $J=1$ and $K \rightarrow \infty$ (as $n \rightarrow \infty)$ recovers the TSRV estimator.

The multi-scale realized volatility (MSRV) estimator [Zhang (2005)] is defined as

$$
\widehat{\langle X, X\rangle_{T}^{(m s r v)}}=\sum_{i=1}^{M} a_{i}[Y, Y]_{T}^{\left(K_{i}\right)}+2 \widehat{\mathbb{E} \epsilon^{2}}
$$

where $M>2$. The weights $a_{i}$ are selected to make the estimator unbiased and to achieve the optimal convergence rate of $n^{-1 / 4}$ (the TSRV has slower convergence rate of $\left.n^{-1 / 6}\right)$. The optimal weights are

$$
a_{i}^{*}=\frac{i}{M^{2}} h^{*}\left(\frac{i}{M}\right)-\frac{i}{2 M^{3}} h^{* \prime}\left(\frac{i}{M}\right),
$$

where $h^{*}(x)=12(x-1 / 2)$ and $h^{* \prime}$ its first derivative. It can be shown that the MSRV is quite robust to the nature of the noise as long as the noise is stationary and sufficiently mixing [see Zhang (2005) and Aït-Sahalia, Mykland, and Zhang (2006)]. 
We next define several signal-to-noise ratios (SNRs) that play a central role in our empirical analysis. We first define $\mathrm{SNR}_{1}$ as

$$
\mathrm{SNR}_{1}:=\frac{T S R V}{R S R V}=\frac{\mathrm{Eq} \cdot(3)}{\mathrm{Eq} \cdot(2)}
$$

i.e., as the ratio of the estimates of true volatility and noise variance. Similarly, we define $\mathrm{SNR}_{2}$ and $\mathrm{SNR}_{3}$ with GTSRV and MSRV in the numerator, respectively.

In theory, noise variance could be estimated unbiasedly by using the negative of the first-lag autocovariance of returns [see, e.g., Roll (1984), Zhou (1996), and Hansen and Lunde (2006)]. It would also be quite robust to jumps [see Oomen (2005b)]. Unfortunately, in practice this "Roll-estimator" can easily produce negative variances due to estimation error [see Harris (1990b)], illiquidity, or positive serial dependence. In order to prevent negative variances, we define $\mathrm{SNR}_{1 b}, \mathrm{SNR}_{2 b}$, and $\mathrm{SNR}_{3 b}$ so that the noise variance is estimated by the absolute value of the first-lag autocovariance. We denote this alternative estimator by $\left|\operatorname{Cov}_{1}\right|$. Notice that it only makes sense to calculate it using trade price data (as explained in the next section).

Because jumps have been shown to have a deteriorating effect not only on the RSRV estimator but also on the TSRV and MSRV estimators [see Fan and Wang (2006)], we consider the impact of jumps in more detail later (see Section 4.3).

\section{Data description}

We use Trades and Quotes (TAQ) data supplied by the NYSE. The decimalization process to cents was completed on January 29, 2001 and we refer to this date as the decimalization date. We analyze two periods of approximately equal length before and after it: November 6, 2000 - January 19, 2001 (the before decimalization period) and February 5, 2001 - April 12, 2001 (the after decimalization period). We exclude one business week on both sides of the decimalization date in order to minimize confounding effects that may arise from the adoption of the new rules. We also exclude one 
abnormally short trading day (Nov/24/2000). This amounts to having 50 and 48 trading days in the before and after period, respectively. Although the data do not span a long time period, there are thousands of observations per day for an active stock which increases our confidence in the empirical results. Focusing on relatively short "before" and "after" periods close to each other also helps to avoid trends.

As is standard in the literature, we consider only NYSE trades and quotes that are time-stamped during the normal trading hours $(9: 30-16: 00 \mathrm{EST})$. We now explicitly want to exclude all other U.S. exchanges such as the Nasdaq in order to minimize noise contamination due to different decimalization schedules and market structures. We exclude trades reported out-of-sequence and quotes that do not correspond to a normal trading environment. ${ }^{7}$ We choose to merge together all simultaneous trades and quotes. The percentage of mergers in the trade data is typically small $(0.5-3 \%)$ but larger in the quote data especially for the most active stocks (up to 30\%). Merging (compressing) the data is a quite common procedure and we do not find it changing the autocorrelation structure significantly. Hansen and Lunde (2006) actually argue it to improve the precision of the volatility estimators.

We form three groups of stocks based primarily on their date of decimalization. In "Control Group 18" (CG18) we include the 18 most active stocks that were decimalized in the first two pilot phases in August and September, 2000. ${ }^{8}$ The rest of the pilot stocks are not active enough for our purposes as we do not want to include stocks from the third pilot phase (December) because it would limit the number of time series observations too much. (All pilot decimalized stocks and their times of decimalization are reported in Appendix A.2). In "Test Group Dow Jones" (TGDJ) we include 30 Dow Jones Industrial Average index stocks that were decimalized on the decimalization date, January 29, 2001 (see Table 1). ${ }^{9}$ They are typically much more active and have

\footnotetext{
${ }^{7}$ More precisely, in the quote data, we keep modes $1,2,3,6,10$, and 12 . In the trade data, we exclude all other trades than the so-called regular trades. See the TAQ2 User Guide for details.

${ }^{8}$ We exclude AOL and TWX from the analysis because of their merger in January, 2001. On the other hand, we keep DCX and UBS although they are American Depositary Receipts because Ahn, Cao, and Choe (1998) find that order flows do not seem to migrate easily from market to market.

${ }^{9}$ Because MSFT and INTC are primarily Nasdaq stocks but part of the Dow Jones Industrial Average index, they are replaced by LU and VZ. Moreover, because of a stock split, JPM is replaced
} 
larger market capitalization than the CG18 stocks. We thus decide to form another test group called "Test Group 18" (TG18) in which we include 18 stocks of similar activity to CG18. In order to improve the match between them, the TG18 stocks are also pairwisely chosen from the same industry subsector or sector as the CG18 stocks (see Table 2). ${ }^{10}$ Some descriptive statistics of all the stocks included in our analysis are reported in Tables 3 (CG18 and TG18) and 4 (TGDJ).

Data errors are likely to be more frequent in UHF data than in sparsely sampled data. With all the trades and quotes at our disposal, however, the errors are also easier to detect. In the terminology of Aït-Sahalia, Mykland, and Zhang (2006), we say that a midquote or trade price is a "bounceback" if a (logarithmic) return larger than a prespecified threshold is followed by a return of similar magnitude but of opposite sign (so that the returns approximately cancel out). We use the following threshold rule: for stocks priced below $\$ 10$, in between $\$ 10$ and $\$ 50$, in between $\$ 50$ and $\$ 100$, and larger than $\$ 100$ we set the threshold to $0.01,0.0083,0.0067$, and 0.005 , respectively. In the before decimalization period, we further multiply the thresholds by 1.5 for stocks trading in fractions (although this adjustment turns out to be insignificant). We find that in general there are more bouncebacks in the trade price data than in the midquote data. This suggests that trade price data are inherently more noisy (bouncy). We delete all found bouncebacks.

Bouncebacks can also be "sticky" in the sense that a data error can repeat for a while. Sticky bouncebacks are much more of a problem in the midquote data than in the trade price data. We detect them by comparing the return and the corresponding spread to the daily standard deviation and spread, respectively. If the spread increases only temporarily from its daily average and is followed by a midquote returning to its previous level, a sticky bounceback is detected and deleted unless it is easy to correct by PFE.

${ }^{10}$ One could match also with respect to other factors such as price, volatility, and equity market capitalization [see, e.g., Chakravarty, Wood, and van Ness (2004) and Chung, van Ness, and van Ness (2004)] but we find our criteria to be adequate. The average prices, for example, are similar between the stock groups (see Tables 3 and 4). In particular, the noise variance estimates of CG18 and TG18 are statistically the same but larger than of TGDJ (as seen in a later table). 
for. ${ }^{11}$ These procedures detect the errors well.

When the sampling frequency gets high, the choice of data type becomes more relevant. It is often argued that a midquote (defined as the average of the bid and ask quotes) provides a less noisy measure of the unobserved true price [see, e.g., Hasbrouck (2007)]. On the NYSE, the variance of the market microstructure noise of midquote returns actually partly reflects the bid-ask quote setting behavior of the specialists. For example, if the price of a stock suddenly moves significantly (up or down), the spread tends to widen momentarily (in the same direction) due to inventory positioning and information asymmetry. In our analysis, we use both trade price and midquote data in order to show how differently decimalization can affect them. ${ }^{12}$ We use the superscripts " $t$ " and " $q$ " to denote their respective estimates.

The key statistical difference between midquotes and trade prices relevant to us is that the first differences of midquotes do not typically have significant negative firstlag autocorrelation commonly addressed to the bid-ask bounce [Roll (1984)], rounding errors [Harris (1990a)], and inventory imbalances [e.g., Jegadeesh and Titman (1995)]. Instead, the midquote returns typically show significant positive autocorrelation for a few lags (and zero afterwards). While this is generally true, the strength of the dependence can vary over time and across stocks [see Hansen and Lunde (2006) and Curci and Corsi (2006)]. We find, for example, that the Ljung-Box (LB) test statistic is able to vary considerably between days and stocks. It tends to be stronger (weaker) in midquote returns (trade price returns) after decimalization (see Tables 3 and 4). We return to this issue in the next section where statistical tests are run. Furthermore, it is not uncommon to observe other sort of autocorrelation patterns, especially for the less active stocks such as the CG18 and TG18 stocks. The autocorrelation structure also depends on the concept of time (the clock).

In our analysis, the clock is set in event time. Event time refers either to trade or

\footnotetext{
${ }^{11} \mathrm{We}$ avoid deleting consecutive quotes because it leaves a gap in the durations between quotes. Deleting consecutive quotes can be especially harmful for an inactive stock. Because it is sometimes hard to diagnose data errors correctly, the remainder becomes part of market microstructure noise.

${ }^{12}$ Naturally other types of data could be used as well, for example weighting the bid and ask quotes by the respective volumes. This would create a more bouncy price process than averaging.
} 
quote time depending on the data type used. It is defined by taking each consecutive event (trade or quote) in consideration with equal weigth so that the distance between two consecutive events is always one unit of time. ${ }^{13}$ This guarantees that no data (information) is thrown away. This gives us an edge over the earlier decimalization studies typically using the "old-school" way of sampling at equidistant calendar time intervals (e.g., $1 \mathrm{~min})$. It is nowadays also widely believed that calendar time sampling is not very well-suited for the analysis of the evolution of a true price in liquid markets [see, e.g., Frijns and Lehnert (2004)]. The problems of calendar time sampling arise from the use of mandatory artificial price construction rules (e.g., interpolation between two prices) and from several well-known intraday patterns which tend to make the calendar time sampled series non-stationary.

\section{Empirical analysis}

\subsection{Preliminary analysis}

We now descriptively evaluate the decimalization effects on volatility and market microstructure noise variance. We also demonstrate how well the non-parametric estimators described above perform. This should facilitate the interpretation of the test results in the next section.

We first find that the TSRV, GTSRV, MSRV, and RSRV estimators adapt quite naturally to different data types and concepts of time. The parametric methods suggested in the literature [see, e.g., Aït-Sahalia, Mykland, and Zhang (2005a)] would not be nearly as flexible. The parametric methods would, in particular, require us to take a stand on the structure and strength of the noise. This would complicate matters considerably in an empirical study like ours where many stocks are analyzed. Although the non-parametric volatility estimators we use are also quite robust to data errors, we

\footnotetext{
${ }^{13}$ This is different from "tick time" with only the instants having a non-zero price change recorded [see, e.g., Oomen (2005a) and Griffin and Oomen (2006)]. Both clocks adapt to the activity of the market but we find the trade and quote time to be more natural.
} 
encourage some attention to be paid to the choice of time-scales that are being averaged over. We next describe a setup which we found reasonable (although our results are not sensitive to this setup).

The TSRV and MSRV estimates are calculated with $K$ and $M$ matching the number of quotes (trades) in 10 or 15 minutes on average, respectively. These choices may at first seem arbitrary and as such to produce considerable amount of estimation error but as Aït-Sahalia, Mykland, and Zhang (2006) have shown, these two estimators are quite robust in this sense. We have here merely tried to adjust the estimators to the daily pace of the market without using any complicated optimality formulas. For very active stocks, obviously, $K$ and $M$ can be much larger than for inactive stocks. Because this may cause problems for the least active stocks on slow days, we fix the lower limit to $K=10$ if there are less than 10 observations in 10 minutes. We do not make any such adjustment to the MSRV estimator. For the GTSRV, we select $J$ according to how strong the daily autocorrelation is: if the LB test statistic is greater than 25 (the chi-square $5 \%$ critical value), then we use $J=2$ and 5 for the trade price and midquote data, respectively. These choices reflect the typical autocorrelation pattern for an active stock. If the daily autocorrelation is weak (LB is less than 25 ), then we use $J=1$ (corresponding to the TSRV). We advice against using a too large $J$ if the strength of the dependence does not call for it because this would cause underestimation.

Tables 5 and 6 report the average $\operatorname{MSRV}^{t}$ (trade price data) estimates for each stock before and after decimalization. The many downward pointing arrows suggest that there is a general tendency for the volatility to be lower after decimalization. These tables also show that although the $\mathrm{MSRV}^{t}$ estimates are close to the $\mathrm{MSRV}^{q}$ (midquote data) estimates, the former are on average around $4 \%$ above the latter regardless of the period and stock group (see columns $\%^{q}$ ). On the other hand, the RVt estimates are clearly inflated before decimalization and tend to become closer to the MSRV ${ }^{t}$ estimates after decimalization (see columns $\%_{r v}^{t}$ ). For TGDJ, for which this effect is particularly evident, the reduction is from $-174 \%$ to $-26 \%$ implying only moderate overestimation compared to the MSRV ${ }^{t}$ estimates after decimalization (see Table 6). In contrast, the 
$\mathrm{RV}^{q}$ estimates are deflated compared to the $\mathrm{MSRV}^{t}$ estimates before decimalization. The noise reduction is also far less obvious than with the trade price data (see columns $\left.\%_{r v}^{q}\right)$. Interestingly, for the inactive stocks the $\mathrm{RV}^{q}$ estimates are actually close to the $\mathrm{MSRV}^{q}$ estimates in both periods (see Table 5).

The TSRV estimates do not show any clear tendency for over or underestimation for the less active stocks (CG18 and TG18). For TGDJ, the TSRV ${ }^{t}$ estimates are again around $4 \%$ above the $\mathrm{TSRV}^{q}$ estimates in both periods. The GTSRV estimates seem to be more sensitive to the activity of the stock. This is probably due to the generic choice of $J$ which works better with the active than the inactive stocks. For example, for TGDJ, the trade price and midquote GTSRV estimates are on average very close to each other (within 1\% margin), but for CG18 and TG18 the GTSRV ${ }^{t}$ estimates tend to be significantly lower than the GTSRV ${ }^{q}$ estimates. (The TSRV and GTSRV estimates are reported in Appendix A.3.)

Figure 1 illustrates how the volatility estimators compare to each other in the case of an active Dow Jones stock, Pfizer Inc. (PFE). The TSRV, GTSRV, and MSRV estimates are close to each other regardless of the data type used. On the other hand, as seen in subplot (a), the $\mathrm{RV}^{t}$ estimates are clearly inflated before the decimalization date but more in line with the others after it. In subplot (b) we see that the $\mathrm{RV}^{q}$ estimates are close to the other estimates in both periods and that the reduction due to decimalization seems much less significant than the corresponding trade price data reduction.

Tables 7 and 8 report the average $\mathrm{RSRV}^{t}$ noise variance estimates. Again we see a tendency for lower estimates after decimalization. The RSRV ${ }^{t}$ estimates are above the $\operatorname{RSRV}^{q}$ estimates especially before decimalization (see columns $\%^{q}$ ). For TGDJ (Table 8), for example, the RSRV ${ }^{t}$ estimates are on average $73 \%$ higher than the $\mathrm{RSRV}^{q}$ estimates before decimalization and $53 \%$ higher after decimalization. Decimalization thus appears to have made the RSRV estimates closer to each other by decreasing the noise more in the trade price data than in the midquote data. That the difference between them stays large can be due to various forms of data dependencies, such as dependence 
(a)

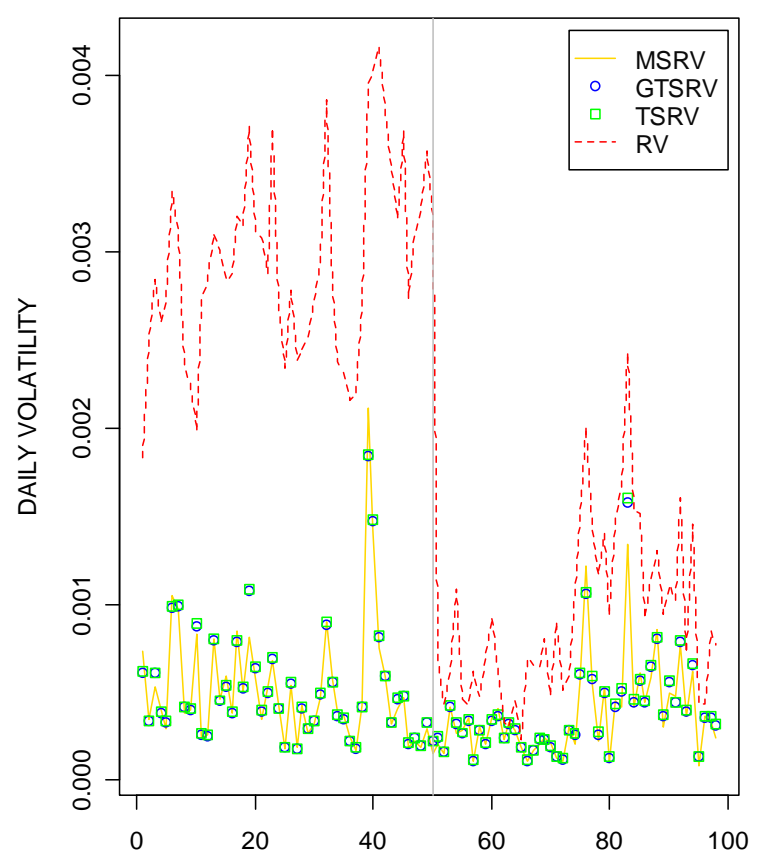

(b)

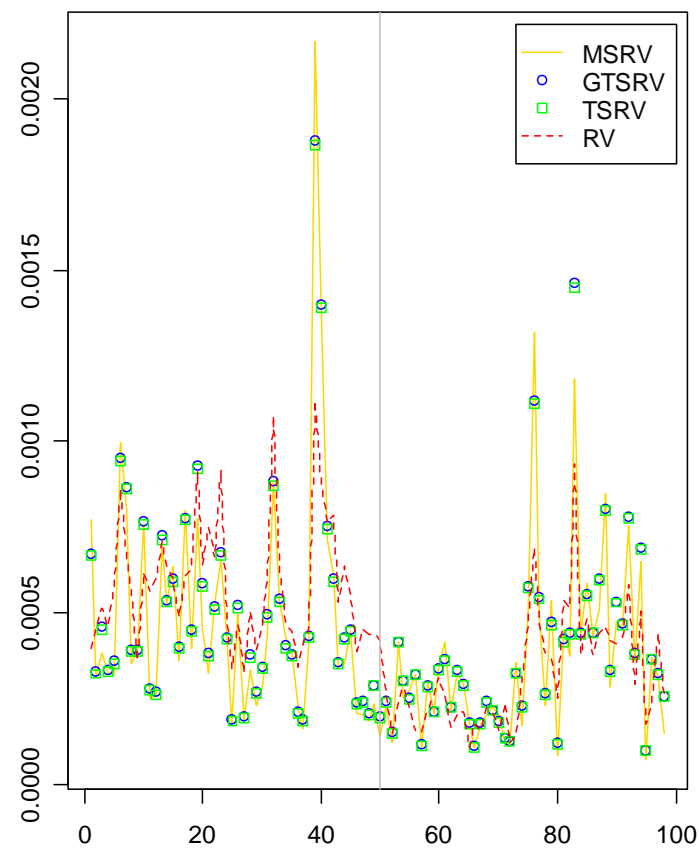

Figure 1: Volatility of PFE using (a) trade prices; (b) midquotes. The last day of the before decimalization period is marked at observation \#50 by the vertical line.

between the noise and the true volatility [see Hansen and Lunde (2006)]. In these two tables we also report the first-lag autocovariance estimates for the noise variance. Except perhaps for TGDJ, however, they are dubious. Some of them actually imply negative variances (the daily estimates even more often so). Thus in the subsequent analysis we mainly rely on the RSRV estimates instead.

Figure 2 illustrates the associated changes in the noise variance and the SNR. The decrease in the $\mathrm{RSRV}^{t}$ estimates and the increase in the $\mathrm{SNR}_{3}^{t}$ are obvious in subplots (a) and (b). The changes are less apparent using the midquotes as can be seen in subplots (c) and (d). In subplots (a) and (b) we have also included the first-lag autocovariance and the corresponding $\mathrm{SNR}_{3 b}^{t}$ estimates. The former are below the RSRV ${ }^{t}$ estimates. In fact, they are close to the $\mathrm{RSRV}^{q}$ estimates, suggesting that the midquotes could be used to reduce the upward biasedness of the trade price data estimates (see Tables 7 and 8). As a consequence, the $\mathrm{SNR}_{3 b}^{t}$ are above the $\mathrm{SNR}_{3}^{t}$ and vary wildly. 
(a)

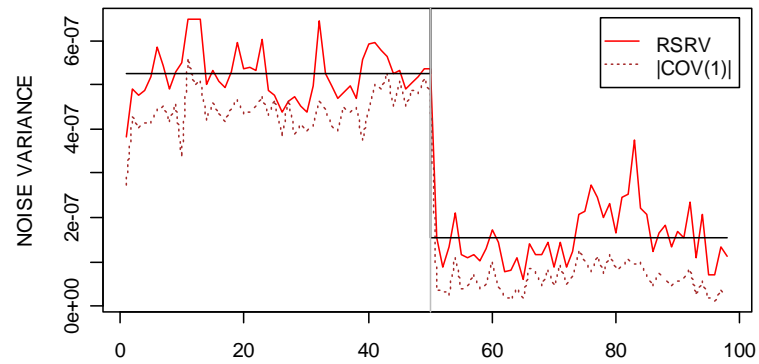

(b)

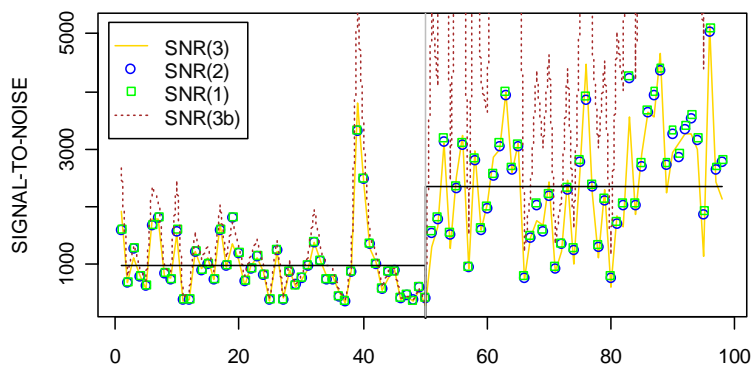

(c)

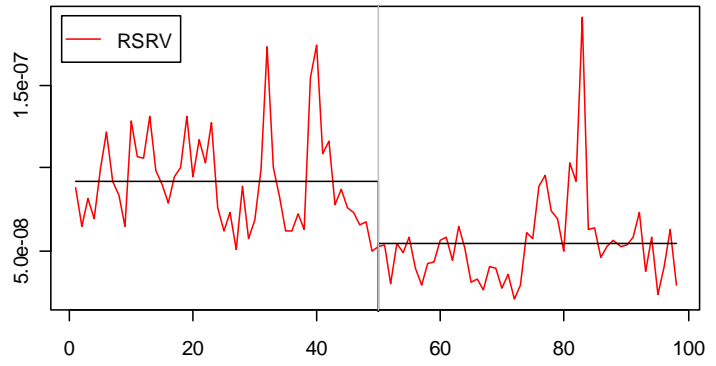

(d)

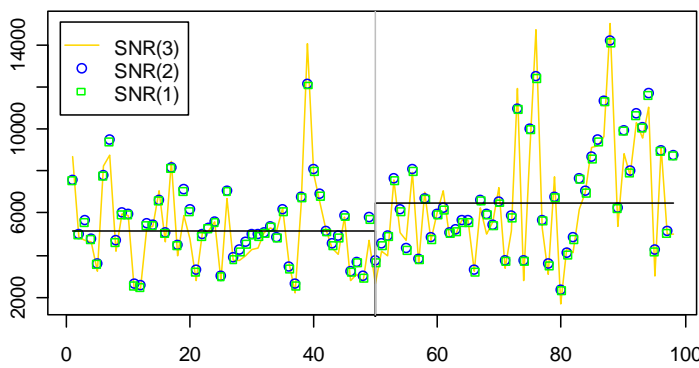

Figure 2: Noise variance and SNR of PFE using trade prices (left panel) and midquotes (right panel). The solid black horizontal lines denote the mean of RSRV (upper panel) and $\mathrm{SNR}_{3}$ (lower panel). The vertical line at observation \#50 denotes the last day of the before decimalization period.

\subsection{The test results}

In this section, which contains the bulk of the results, we use the paired $t$-test for a group of non-independent samples in order to test whether the means of various variables were different from each other before and after the final decimalization date. The paired $t$-test is standardly applied in similar contexts including a few earlier decimalization studies [see, e.g., Bessembinder (2003)]. The test assumes that the differences between the paired values are randomly drawn from the source population and that the source population can be reasonably supposed to be normally distributed. However, because normality of the differences is sometimes a too restrictive assumption, we also use another standard test, namely the Wilcoxon rank sign test, which requires only symmetry and not normality. The Wilcoxon rank sign test ranks the median values of absolute differences and for large enough samples it is normally distributed [for details, 
see, e.g., Hollander and Wolfe (1999)]. ${ }^{14}$ Notice that serial dependence between observations (i.e., order) in the before and after decimalization periods is not relevant for the calculation of these test statistics because only the differences between the unconditional means are used. And although the assumption of independence between the pairs of stocks may be questioned (especially during big stock market downturns), we regard it is as a reasonable assumption if the stocks are picked in random from different sectors as they essentially now are (see Section 3). Also, the stocks within each group CG18, TG18, and TGDJ are much alike in trading activity which makes taking averages over them less of a problem than if they would not have anything in common.

We first report the paired $t$-test and the Wilcoxon rank sign test results for the absolute and relative spread, number of quotes and trades, quoted depth, trading volume, and serial dependence. This helps us to interpret the main results on volatility and market microstructure noise variance (reported next). Our findings can be compared to the earlier studies with some precautions in mind, mostly related to different market mechanisms (see Section 1).

From Table 9 we see that, for CG18, none of the seven variables change significantly except the number of quotes which increases over the two periods (not abruptly but smoothly). In particular, we find no significant changes in the number of trades, trading volume (in round-lots), or LB test statistics (for 15 lags). Thus the control group is largely unaffected by the decimalization of the other stocks as it ideally should. In contrast, most of the variables for TG18 and TGDJ change significantly. As expected, we find a significant decrease in their quoted depth and a very significant decrease in their spreads. TGDJ has no significant increase in the number of quotes but TG18 does. This is probably due to the fact that the quotes for the most active stocks were already updated frequently before the decimalization date. Interestingly, for both TG18 and

\footnotetext{
${ }^{14}$ The paired $t$-test is calculated as $(\bar{X}-\bar{Y}) /$ S.E. $[(\bar{X}-\bar{Y})]$, where the denominator is the standard error of the difference of the two averages. Notice that the nominator is numerically equivalent with calculating the average of the paired differences. The Wilcoxon rank sum test is calculated as $\sum_{i=1}^{n} R_{i} \psi_{i}$, where $R_{i}$ is the rank of the absolute value of the difference between the before and after period observations and $\psi_{i}$ is an indicator function taking the value 0 when the difference is negative and 1 when the difference is positive.
} 
TGDJ, the midquote return autocorrelation tends to increase. In contrast, the trade return data autocorrelation decreases, although both groups have a significant increase in the number of trades. The decrease is more significant for TGDJ, again probably due to its higher activity. A decrease in the first-lag autocorrelation is in line with theory that predicts less significant first-lag autocorrelation due to smaller rounding error [see Harris (1990a)]. That the number of trades increases for both TG18 and TGDJ while their trading volume stays constant is also in line with the earlier studies.

From Table 10, on the other hand, we see that the midquote absolute first-lag covariances decrease or do not change at all. Because the corresponding LB test statistic was just found to increase (see Table 9), decimalization seems to have weakened the first-lag dependence but strenghtened the higher lag dependence. This would explain the apparent controversy between the LB of midquote and trade data (although this is stock dependent to some extent as noted above). The main reason why the dependence should be increased in the midquote data is, we believe, that decimalization changed the strategic behavior of the market participants. The participants outside the "floor" of the NYSE may have for example become more cautious due to the fear of "front-running" (a form insider trading from the part of the NYSE specialists). This is consistent with the reported decrease in market transparency [see, e.g., NYSE (2001)]. In general, the simple and significantly weakened serial dependence in the trade price data should make them better suited for the analysis of decimalization effects than the midquote data especially with the most active stocks.

We now turn to our main results that are reported in Table 10. According to the paired $t$-test, true volatility appears to decrease only slightly (CG18 and TGDJ) or not significantly (TG18). The Wilcoxon test provides much stronger evidence of a decrease which is evidently due to the non-Gaussian character of volatility. The results also reveal that there are in general only minor differences between the three volatility estimators we use. The MSRV estimates indicate a slightly more significant decrease for TGDJ (whether this may be due to jumps is discussed in the next section). Noise variance estimates decrease significantly across the board. For TG18 and TGDJ, the 
decrease in noise variance turns out to be more significant than the volatility decrease. Not surprisingly, then, the increase in their SNR is highly significant for TGDJ. We find no major differences between $\mathrm{SNR}_{1}, \mathrm{SNR}_{2}$, and $\mathrm{SNR}_{3}$ but $\mathrm{SNR}_{3}$ changes slightly less significantly than the others.

Notice that the results using trade price data (left panel in Table 10) differ in some respects from the midquote data results (right panel). The trade price data tend to give slightly more significant results than the midquote data for the volatility of TGDJ. The difference is more obvious for the noise variance. As a consequence, the increase in $\mathrm{SNR}^{t}$ for TGDJ turns out to be highly significant. The data type does not seem to matter as much for TG18, however, suggesting that the less active stocks are less affected by the data type.

It is of course possible that the tests applied here accidentally capture inherent trends in the data and, in the case of volatility in particular, clustering to turbulent and tranquil times that are not related to the decimalization process itself. As a first attempt to control for confounding factors, we run linear regressions for each stock with volatility, noise variance, and SNR as the dependent variables. The explanatory variables we use are the number of quotes (for the midquote data) and trades (for the trade price data), the LB test statistic, and a dummy variable defined to be 0 before decimalization and 1 after it. The number of quotes and trades are included because they appear prominently in the calculation of the estimates (see Section 2) and because they have not, in general, remained constant over the sample period (see Table 9). The LB test statistic, on the other hand, is included because it can be thought as reflecting the daily information asymmetry and because it has not in general remained constant either. Note that we do not include the spreads as explanatory variables because they correlate highly with the dummy variable for the TGDJ stocks. Multicollinearity could easily lead to invalid inference regarding the regression coefficients. Such approximate multicollinearity actually only supports the view that the most active stocks had the largest spread and noise variance reductions.

The stock specific regressions indicate that the dummy variable tends to be signif- 
icantly negative when the dependent variable is either volatility or noise variance. So confounding factors do not appear to be responsible for the decrease. It is however harder to tell what the effect on the SNR is because the corresponding dummy coefficient is often close to zero and its sign alternates stock dependently within and between groups. In order to gain more insight, we run panel (longitudinal) linear regressions with the same dependent and explanatory variables. Specifically, we use the restricted maximum likelihood method to estimate linear mixed-effects models of the form [see Pinheiro and Bates (2004, Ch. 2)]

$$
\begin{aligned}
y_{i t}= & \alpha+\beta_{1} L B(15)_{i t}+\beta_{2} N r \text { Trades }(\text { Quotes })_{i t}+\beta_{3} \text { Dummy } y_{i t}+ \\
& a_{i}+b_{1 i} L B(15)_{i t}+b_{2 i} N r \text { Trades }(\text { Quotes })_{i t}+b_{3 i} \text { Dummy } y_{i t}+\epsilon_{i t},
\end{aligned}
$$

where $i$ denotes the $i$ th stock in CG18/TG18 $(i=1, \ldots, 18)$ or TGDJ $(i=1, \ldots, 30)$ and $t$ denotes the day before and after decimalization $(t=1, \ldots, 98)$. Here $\alpha$ and $\beta_{h}$ (on the top row) are the fixed-effect coefficients and $a_{i}$ and $b_{h i}$ (on the bottom row) are the random-effect coefficients. The latter describe the shift in the intercept and the explanatory variables for each stock, respectively, and they are assumed to be normally distributed with mean zero. The random-effect coefficients are allowed to be correlated (with a covariance matrix not depending on the stock) but the normally distributed and zero-centered error terms $\epsilon_{i t}$ are assumed to be independent. Thus, the random effects can be regarded as additional errors terms that account for correlation among observations (for each stock separately).

The trade price and midquote data panel regression results are reported in Tables 11 and 12. In them, volatility is estimated by the MSRV (the other estimators would do as well). In order to facilitate interpretation of the regression coefficients and to mitigate multicollinearity, we center the explanatory variables except the dummy. As seen above, we include random effects for all the explanatory variables including the dummy. This tends to give less significant fixed-effect regression coefficients for the dummy. Despite the more conservative inference, the coefficients for the dummy are negative and clearly 
significant when volatility and noise variance are dependent variables. However, there does not appear to be much support for a significant abrupt increase in the SNR: after controlling for the number of quotes and trades, in particular, the coefficient for the dummy turns out to be insignificant or of the wrong sign (midquote data) or to be only mildly significant (trade price data). Specification tests done along the lines of Pinheiro and Bates (2004, Ch. 4.3) do not reveal any clear evidence of misspecifation - especially when random effects are included for every fixed-effect explanatory variable as they now are. In particular, we then find that the standardized within-stock residuals are zero centered and normally distributed.

We conclude that after taking confounding and random effects into account, at best only weakly significant evidence of an abrupt level shift in the SNR exists and that only the trade price data provide evidence in favor of an increase. The last point makes sense because the trade price data are more sensitive to the change in the tick size. In fact, if the midquotes correctly represent the true price, the corresponding dummy should not be significant. Of course, we cannot totally exclude the possibility that the above findings are not due to the relatively short sample period in question. However, longer periods or periods farther apart from each other would potentially suffer even more from trends and complicate the inference.

\subsection{The effect of jumps}

It is a well-known theoretical fact that if jumps exist, RV does not converge to IV even in the absence of market microstructure noise but instead to $I V+\sum_{0 \leq t \leq T}\left|\Delta X_{t}\right|^{2}$, where $\Delta X$ denotes a large return (a true jump triggered by an earnings announcement, for example). It has also been reported that estimators such as the TSRV and MSRV then lose part of their estimation accuracy [see Fan and Wang (2006)]. This has raised some doubts about the validity of earlier research [see, e.g., Diebold (2005)]. We thus next study if jumps exist in our data and if the jumps affect our results on decimalization effects significantly. Because there is some ambiguity between jumps and noise in 
discrete data, we also pay some attention to how much noise there is in the trade price data compared to the midquote data.

We first test for the presence of jumps. For this purpose we use the test proposed by Aït-Sahalia and Jacod (2006) which we call the two-scale jump test (TSJT). This test is valid only in the absence of market microstructure noise so we expect to see some bias, but we nevertheless find it useful to calculate for qualitative purposes. The good thing is that the TSJT is a particularly direct and easy way to test for the presence of jumps (but not their strength or frequency) without making strong assumptions. We now shortly review it.

The TSJT statistic is defined as

$$
\widehat{S}\left(p, k, \Delta_{n}\right)_{t}=\frac{\widehat{B}\left(p, k \Delta_{n}\right)_{t}}{\widehat{B}\left(p, \Delta_{n}\right)_{t}}
$$

where, for $p>0$,

$$
\widehat{B}\left(p, \Delta_{n}\right)_{t}:=\sum_{i=1}^{\left[t / \Delta_{n}\right]}\left|\Delta_{i}^{n} X\right|^{p}
$$

is the estimator of variability, and $\Delta_{i}^{n} X=X_{i \Delta_{n}}-X_{(i-1) \Delta_{n}}$ denote the discrete increments of a semimartingale process $X$. As is easily observed, the TSJT statistic takes advantage of two overlapping time-scales, $\Delta_{n}$ and $k \Delta_{n}$, from which we have derived its name.

The usefulness of this test is based on a theorem in Aït-Sahalia and Jacod (2006) saying that if $t>0, p>2$, and $k \geq 2$, then the variables $\widehat{S}\left(p, k, \Delta_{n}\right)_{t}$ converge (in probability) to the variable $S(p, k)_{t}=1$ on the set of discontinuous processes and to $k^{p / 2-1}$ on the set of continuous processes as $\Delta_{n} \rightarrow 0(n \rightarrow \infty)$. Aït-Sahalia and Jacod (2006) show that if the market microstructure noise is IID and $p=4$, then the TSJT statistic converges to $1 / k$ (instead of 1$)$. They note, however, that when $\mathbb{E} \epsilon^{2}$ and $\mathbb{E} \epsilon^{4}$ are small (as in practice they are; see for example $\widehat{\mathbb{E} \epsilon^{2}}$ in Tables 7 and 8) and $\Delta_{n}=T / n$ is "moderately small," then the TSJT statistic will again be close to 1 in the discontinuous case. 
We use the values $p=4$ and $k=2$ and set $[0, T]$ to one trading day as suggested by Aït-Sahalia and Jacod (2006). We let $\Delta_{n}$ to vary from 1 to 10 events but report here only the results with $\Delta_{n}=1$ (the larger time-scales give less accurate results). The non-normalized histograms of all the stocks together are presented in Figure 3. There are 6468 observations in total, each observation representing one day for one stock. The subplots (a) and (b) indicate that market microstructure noise is prominent because the histograms are not tightly peaked around any value. In particular, the histograms are not centered around 1 or 2 . The mean of the midquote data is higher than the mean of the trade price data. This is in line with the view that the midquote data are less bouncy than the trade price data (see Section 3). ${ }^{15}$

For comparison purposes, in subplots (c) and (d) we show the histograms in calendar time with $\Delta_{n}=5$ seconds. These histograms are more sharply centered around 1, implying more jumpy price evolution or less noise contamination in calendar time. Both explanations are in fact plausible. ${ }^{16}$ The price process may be more jumpy in calendar time because the price does not adjust instantly to new surprising news. This would be consistent for example with the idea of subordination and the directing process evolving at different rates [see, e.g., Clark (1973)]. In event time the jumps also tend to be smoothed out by market makers who monitor the market closely. The fact that the histograms of trade price and midquote data look more like each other in calendar than in event time supports the view that the data type plays a less significant role in larger time-scales (and with less active stocks).

There are at least two straightforward ways to remove jumps [see Barndorff-Nielsen and Shephard (2004) and Aït-Sahalia and Jacod (2006)]. Both of these methods (as they currently stand), however, work well only in the absence of market microstructure noise. Thus we decide to apply the following simple rule instead: if the return of consecutive prices in event time is larger than a given threshold (we use 0.03), then the

\footnotetext{
${ }^{15}$ It would not make sense to call the trade price data truly more jumpy than the midquote data because both data types reflect the same underlying value.

${ }^{16}$ Aït-Sahalia and Jacod (2006) find their histogram to be centered below 1 . They however include Nasdaq data in their analysis which increases the amount of noise and explains the shift in location.
} 
(a)

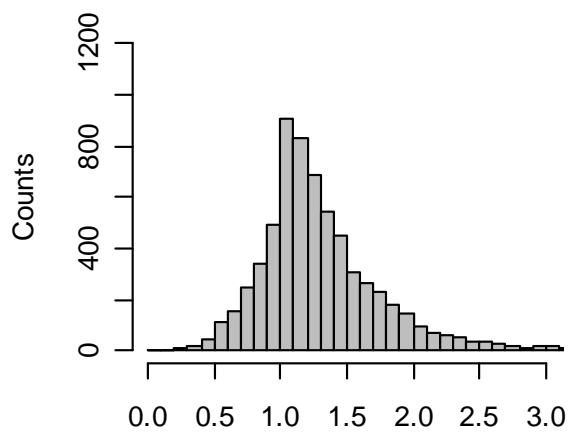

Mean (trade time): 1.312

(b)

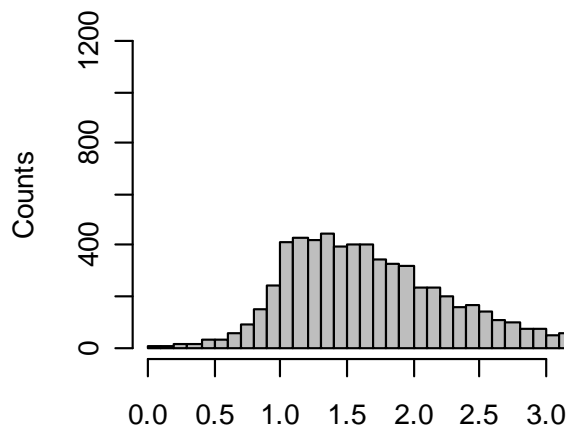

Mean (quote time): 1.772 (c)

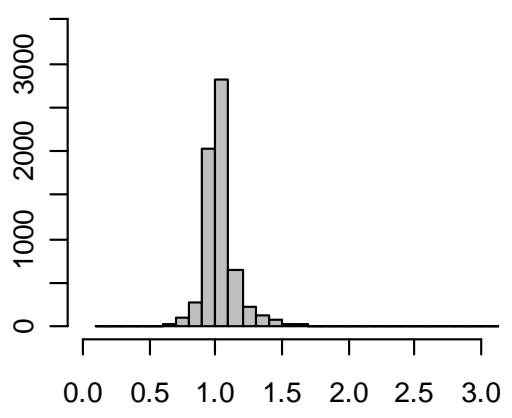

Mean (calendar time): 1.043

(d)

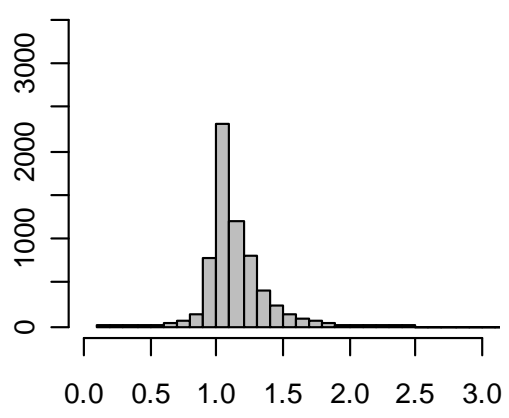

Mean (calendar time): 1.149

Figure 3: Histograms of the TSJT statistic in event time using (a) trade prices; (b) midquotes. Subplots (c) and (d) show the corresponding data in calendar time.

return is removed, a new price table is constructed, and the statistical tests are re-run. This reduces the daily number of quotes and trades but only slightly because so big jumps are quite rare in event time. In fact, there are only a few such jumps in each group per period and less in the midquote data than in the trade price data. Of course, the number of jumps would be greatly increased if we would use a smaller threshold but then we would also be more likely to capture not only the true jumps but also noise. This would be especially true with the less active CG18 and TG18 stocks. For completeness, we report the number of jumps with three different thresholds in Tables 13 and 14 which clearly show the increase in the number of jumps when the threshold is decreased $(0.03 \rightarrow 0.01)$. 
Because most of the days do not have any significant jumps according to the above threshold (0.03), the sums of power 4 stay largely unaffected when the jumps are removed. We see no obvious differences between the before and after period either. The paired $t$-test statistics are only moderately affected by the jumps (and the Wilcoxon test less so). The change is most evident for volatility and noise variance; in the case of a low priced stock, a big price swing can make jumps more likely in one of the periods and thus produce many jumps (see, e.g., LU in Table 4). But even in this case the effect on the SNR is diminishingly small because both the noise variance and the true volatility decrease by approximately the same amount. So we conclude that jumps do not seem to have any qualitatively important impact on our results. This seems like a natural result as true jumps are basicly randomly scattered over time independently of the decimalization process and the test statistics we use group many practically independent (although similar in trading activity) stocks together.

\section{Conclusions}

In this paper we have empirically studied the effect of decimalization on volatility and market microstructure noise using UHF data. A key point is to estimate the true volatility accurately. To this end, we have used three non-parametric estimators that all have desirable statistical properties and are yet flexible and simple to calculate. We have estimated the market microstructure noise variance non-parametrically as well. Statistical tests are run in order to evaluate the significance of the effects on volatility, noise variance, and their ratio.

The main result of this empirical study is that decimalization decreased observed volatility by reducing noise variance especially for the highly active stocks. The reduction can be attributed to smoother price evolution or, in other words, to diminished price discreteness. Consequently, the significance of the true signal appears to have increased. Mixed-effect panel regression results show, however, that most of this increase is explainable by confounding and random effects. The trade data give more favorable 
results than the midquote data which is in line with the view that trade prices are more sensitive to the changes in the tick size and that they provide a less accurate estimate of the true (unobserved) price. For the inactive stocks the difference between the two data types appears to be insignificant.

It should be noted, however, that the decrease in observed volatility due to decimalization can be slightly deceptive. As the markets also became less transparent and the market making costs increased, algorithmic trading gained more popularity in so much that it nowadays makes up a big portion of the daily volume. In a crisis, algorithmic trading can lead to sell-off pressures that may actually end up increasing volatility in the decimal regime.

This study also demonstrates how the TSRV, GTRSV, and MSRV estimators perform in a changing environment. The MSRV estimator appears to give the most robust estimates with respect to the data type and tick size used. This is noteworthy because we find that decimalization decreased linear dependence in the trade data but increased it in the midquote data. On the other hand, the MSRV trade price data estimates are on average a few percents higher than the respective midquote data estimates in both periods. This discrepancy may be due the fact that the MSRV estimator does not adjusting to all complex dependencies. Nevertheless, we feel that the non-parametric volatility estimators used here can be considered as more flexible than the parametric estimators suggested in the literature.

Although the estimators we use are sensitive to jumps to certain extent (the volatility estimators less than the RSRV), we do not find true jumps to be critical for our results. Prefiltering the data for errors seems far more important because compared to jumps triggered by news, the errors tend to be bigger, more frequent, more systematic, and harder to correct for especially in the quote data.

Finally, we note that the estimators we have used are accurate only in ideal conditions that may not exist in practice over time and across stocks. We feel that there is room for improvement especially in the estimation of noise variance by taking more complex data dependencies into account. It would also be useful to be able to decom- 
pose volatility into even smaller components. Separating small components from each other would make the effect of decimalization even more transparent. Decomposition of market microstucture noise would however require us to take a stand on questions such as what constitutes a jump and how to separate it from other noise sources. This is a topic of both theoretical and practical interest and likely to become more important as infinitely active jump processes gain more popularity in stock price modeling. We leave these issues for future research. 


\section{References}

Aït-Sahalia, Yacine, and Jean Jacod. (2006). "Testing for Jumps in a Discretely Observed Process." Working Paper, Princeton University and UPMC.

Aït-Sahalia, Yacine, Per A. Mykland, and Lan Zhang. (2005a). "How Often to Sample a Continuous-Time Process in the Presence of Market Microstructure Noise." Review of Financial Studies 18, 351-416.

_ (2005b). "Comment on 'Realized Variance and Market Microstructure Noise' by Peter Hansen and Asger Lunde." Technical Report, University of Chicago.

(2006). "Ultra High Frequency Volatility Estimation with Dependent Microstructure Noise." Working Paper, Princeton University, University of Chicago, and University of Illinois.

Ahn, Hee-Joon, Charles Q. Cao, and Hyuk Choe. (1996). "Tick Size, Spread, and Volume." Journal of Financial Intermediation 5, 2-22.

(1998). "Decimalization and Competition Among Stock Markets: Evidence from the Toronto Stock Exchange Cross-Listed Securities." Journal of Financial Markets 1, $51-87$.

Amihud, Yakov, and Haim Mendelson. (1987). "Trading Mechanisms and Stock Returns: An Empirical Investigation." Journal of Finance 42, 533-553.

Bacidore, Jeffrey M. (1997). "The Impact of Decimalization on Market Quality: An Empirical Investigation of the Toronto Stock Exchange." Journal of Financial Intermediation 6, 92-120.

Bacidore, Jeff, Robert Battalio, and Robert Jennings. (2001). "Changes in Order Characteristics, Displayed Liquidity, and Execution Quality on the New York Stock Exchange Around the Switch to Decimal Pricing." Working Paper, New York Stock Exchange.

(2003). "Order Submission Strategies, Liquidity Supply, and Trading in Pennies on the New York Stock Exchange." Journal of Financial Markets 6, 337-362.

Bandi, Federico M., and Jeffrey R. Russell. (2003). "Microstructure Noise, Realized Volatility, and Optimal Sampling." Working Paper, University of Chicago.

_ (2006). "Separating Microstructure Noise from Volatility." Journal of Financial Economics 79, 655-692.

Barndorff-Nielsen, Ole E., and Neil Shephard. (2004). "Power and Bipower Variation with Stochastic Volatility and Jumps." Journal of Econometrics 2, 1-37.

Bessembinder, Hendrik. (2003). "Trade Execution Costs and Market Quality after Decimalization." Journal of Financial and Quantitative Analysis 38, 747-778.

Black, Fischer. (1986). "Noise." Journal of Finance 41, 529-543. 
Bollen, Nicolas P. B., and Robert E. Whaley. (1998). "Are Teenies' Better?" Journal of Portfolio Management 25, 10-24.

Chakravarty, Sugato, Robert A. Wood, and Robert A. van Ness. (2004). "Decimals and Liquidity: A Study of the NYSE." Journal of Financial Research 27, 75-94.

Chakravarty, Sugato, Venkatesh Panchapagesan, and Robert A. Wood. (2005). "Did Decimalization Hurt Institutional Investors?" Journal of Financial Markets 8, 400-420.

Chakravarty, Sugato, Bonnie F. van Ness, and Robert A. van Ness. (2005). "The Effect of Decimalization on Trade Size and Adverse Selection Costs." Journal of Business Finance and Accounting 32, 1063-1081.

Cho, D. Chinhyung, and Edward W. Frees. (1988). "Estimating the Volatility of Discrete Stock Prices." Journal of Finance 43, 451-466.

Chung, Kee H., Bonnie F. van Ness, and Robert A. van Ness. (2004). "Trading Costs and Quote Clustering on the NYSE and NASDAQ After Decimalization." Journal of Financial Research XXVII, 309-328.

Clark, Peter K. (1973). "A Subordinated Stochastic Process Model with Finite Variance for Speculative Prices." Econometrica 41, 135-155.

Curci, Giuseppe, and Fulvio Corsi. (2006). "Discrete Sine Transform for Multi-Scales Realized Volatility Measures." Working Paper, Universita di Pisa and University of Lugano. Available at SSRN: http://ssrn.com/abstract $=650504$.

Diebold, Francis X. (2005). "On Market Microstructure Noise and Realized Volatility." Working Paper, University of Pennsylvania.

Easley, David, and Maureen O'Hara. (1992). "Time and the Process of Security Price Adjustment." Journal of Finance 47, 577-605.

Economist. (2007a). "Dodgy Tickers; Stock Exchanges." Economist, March 10, 2007.

Economist. (2007b). "Ahead of the Tape; Algorithmic Trading." Economist, June 23, 2007.

Engle, Robert F., and Zheng Sun. (2005). "Forecasting Volatility Using Tick by Tick Data." Working Paper, New York University. Available at SSRN: http://ssrn.com/abstract $=676462$.

Fan, Jianqing, and Yazhen Wang. (2006). "Multi-Scale Jump and Volatility Analysis for High-Frequency Financial Data." Working Paper, Princeton University and University of Connecticut.

Figlewski, Stephen. (1997). "Forecasting Volatility." Financial Markets, Institutions, and Instruments 6, 1-88.

Frijns, Bart, and Thorsten Lehnert. (2004). "Realized Variance in the Presence of NonIID Microstructure Noise." Working Paper, Maastricht University. 
Gibson, Scott, Rajdeep Singh, and Vijay Yerramilli. (2003). "The Effect of Decimalization on the Components of the Bid-Ask Spread." Journal of Financial Intermediation $12,121-148$.

Goldstein, Michael A., and Kenneth A. Kavajecz. (2000). "Eigths, Sixteenths, and Market Depth: Changes in Tick Size and Liquidity Provision on the NYSE." Journal of Financial Economics 56, 125-149.

Gottlieb, Gary, and Avner Kalay. (1985). "Implications of the Discreteness of Observed Stock Prices." Journal of Finance 40, 135-153.

Glosten, Lawrence R., and Paul R. Milgrom. (1985). "Bid, Ask, and Transaction Prices in a Specialist Market with Heterogenously Informed Traders." Journal of Financial Economics 14, 71-100.

Griffin, Jim E., and Roel C. A. Oomen. (2006). "Sampling Returns for Realized Variance Calculations: Tick Time or Transaction Time?" Working Paper, University of Warwick. Available at SSRN: http://ssrn.com/abstract $=906472$.

Hansen, Peter R., and Asger Lunde. (2006). "Realized Variance and Market Microstructure Noise." Journal of Business and Economic Statistics 24, 127-161.

Harris, Lawrence. (1990a). "Estimation of Stock Price Variances and Serial Correlations from Discrete Observations." Journal of Financial and Quantitative Analysis 25, 291306.

(1990b). "Statistical Properties of the Roll Serial Covariance Bid/Ask Spread Estimator." Journal of Finance 45, 579-590.

— (1991). "Stock Price Clustering and Discreteness." Review of Financial Studies 4, $389-415$.

(1994). "Minimum Price Variations, Discrete Bid-Ask Spreads, and Quotation Sizes." Review of Financial Studies 7, 149-178.

— (1997). "Decimalization: A Review of the Arguments and Evidence." Working Paper, University of Southern California.

— (1999). "Trading in Pennies: A Survey of the Issues." Working Paper, University of Southern California.

Hasbrouck, Joel. (1996). "Modeling Market Microstructure Time Series." In G. Maddala, and C. Rao (eds.), Handbook of Statistics 14, North Holland, Amsterdam, pp. $647-692$.

- (2007). Empirical Market Microstructure Theory: The Institutions, Economics, and Econometrics of Securities Trading. Oxford University Press.

He, Yan, and Chunchi Wu. (2005). "The Effects of Decimalization on Return Volatility Components, Serial Correlation, and Trading Costs." Journal of Financial Research XXVIII, 77-96. 
Hendershott, Terrence, Charles M. Jones, and Albert J. Menkveld. (2008). "Does Algorithmic Trading Improve Liquidity?" Available at SSRN: http://ssrn.com/abstract=1100635.

Hollander, Myles, and Douglas A. Wolfe. (1999). Nonparametric Statistical Methods. Second Edition. John Wiley \& Sons.

Huang, Roger D., and Hans R. Stoll. (2001). "Tick Size, Bid-Ask Spreads, and Market Structure." Journal of Financial and Quantitative Analysis 36, 503-522.

Ikenberry, David, and James P. Weston. (2003). "Clustering in U.S. Stock Prices After Decimalization." Working Paper, University of Illinois and Rice University.

Jegadeesh, Narasimhan, and Sheridan Titman. (1995). "Short-Horizon Return Reversals and the Bid-Ask Spread." Journal of Financial Intermediation 4, 116-132.

Jones, Charles M., and Marc L. Lipson. (2001). "Sixteenths: Direct Evidence on Institutional Trading Costs." Journal of Financial Economics 59, 253-278.

Madhavan, Ananth, Matthew Richardon, and Mark Roomans. (1997). "Why Do Securities Prices Change? A Transaction-Level Analysis of NYSE Stocks." Review of Financial Studies 10, 1035-1064.

New York Stock Exchange. (2001). "Decimalization of Trading on the New York Stock Exchange: A Report to the Securities and Exchange Commission." Working Paper, New York Stock Exchange.

Oomen, Roel C. A. (2005a). "Properties of Bias-Corrected Realized Variance Under Alternative Sampling Schemes." Journal of Financial Econometrics 3, 555-577.

— (2005b). "Comment on 'Realized Variance and Market Microstructure Noise' by Peter R. Hansen and Asger Lunde." Journal of Business and Economic Statistics 24, 195-202.

Pinheiro, José C., and Douglas M. Bates. (2004). Mixed-Effects Models in S and S-Plus. Springer.

Porter, David C., and Daniel G. Weaver. (1997). "Tick Size and Market Quality." Financial Management 26, 5-26.

Protter, Philip E. (2005). Stochastic Integration and Differential Equations. Second Edition. Springer.

Ricker, Jeffrey P. (1998). "Breaking the Eight: Sixteenths on the New York Stock Exchange." Available at SSRN: http://ssrn.com/abstract=79759.

Roll, Richard. (1984). "A Simple Implicit Measure of the Bid-Ask Spread in an Efficient Market." Journal of Finance 39, 1127-1139.

Ronen, Tavy, and Daniel G. Weaver. (2001). "Teenies' Anyone?" Journal of Financial Markets 4, 231-260. 
van Ness, Bonnie F., Robert A. van Ness, and Stephen W. Pruitt (1999). "The Impact of the Reduction in Tick Increment in Major U.S. Markets on Spreads, Depth, and Volatility." Review of Quantitative Finance and Accounting 15, 153-167.

Zhang, Lan. (2005). "Efficient Estimation of Stochastic Volatility Using Noisy Observations: A Multi-Scale Approach." Working Paper, University of Illinois and Carnegie Mellon University. Available at SSRN: http://ssrn.com/abstract=619682.

Zhang, Lan, Per A. Mykland, and Yacine Aït-Sahalia. (2005). "A Tale of Two Time Scales: Determining Integrated Volatility with Noisy High-Frequency Data." Journal of the American Statistical Association 100, 1394-1411.

Zhao, Xin, and Kee H. Chung. (2006). "Decimal Pricing and Information-Based Trading: Tick Size and Informational Efficiency of Asset Price." Journal of Business Finance and Accounting 33, 753-766.

Zhou, Bin. (1996). "High-Frequency Data and Volatility in Foreign-Exchange Rates." Journal of Business and Economic Statistics 14, 45-52. 
Table 1: Test Group Dow Jones (TGDJ).

\begin{tabular}{cl}
\hline \hline Ticker & Stock \\
\hline AA & Alcoa Inc \\
AIG & American International Group Inc \\
AXP & American Express Co \\
BA & Boeing Co \\
C & Citigroup \\
CAT & Caterpillar Inc \\
DD & E. I. du Pont de Nemours and Co \\
DIS & Walt Disney Co \\
EK & Eastman Kodak Co \\
GE & General Electric Corp \\
GM & General Motors Corp \\
HD & Home Depot Inc \\
HON & Honeywell International Inc \\
HWP & Hewlett-Packard Co \\
IBM & International Business Machines Corp \\
IP & International Paper Co \\
JNJ & Johnson and Johnson \\
KO & Coca-Cola Co \\
LU & Lucent Techs Inc \\
MCD & McDonald's Corp \\
MMM & Minnesota Mng Mfg Co \\
MO & Altria Group Inc \\
MRK & Merck \& Co Inc \\
PFE & Pfizer Inc \\
PG & Procter \& Gamble Co \\
SBC & SBC Communications Inc \\
T & AT\&T Inc \\
VZ & Verizon Communications \\
WMT & Wal-Mart Stores Inc \\
XOM & Exxon Mobil Co \\
\hline &
\end{tabular}


Table 2: Control Group 18 (left) and the matched Test Group 18 (right).

\begin{tabular}{cl|cl}
\hline \hline Ticker & Stock & Ticker & Stock \\
\hline APC & Anadarko Petroleum Corp & APA & Apache Corp \\
BEN & Franklin Resources & BSC & Bear Stearns Companies Inc \\
CI & Cigna Corp & WLP & Wellpoint Hlth Netwks Hldg Co \\
CL & Colgate-Palmolive Corp & AVP & Avon Products Inc \\
CPQ & Compaq Computer Corp & EMC & EMC Corp \\
DCX & DaimlerChrysler AG Ord & HDI & Harley Davidson Inc \\
FDX & Fedex Corp & UPS & United Parcel Service Inc \\
GMH & General Motors Corp New & F & Ford Motor Co New \\
GT & Goodyear Tire Rubber Corp & CTB & Cooper Tire Rubber Co \\
GTW & Gateway Inc & LXK & Lexmark Intl Inc \\
H & Harcourt General Inc & AM & American Greetings Corp \\
IOM & Iomega Corp & DBD & Diebold Inc \\
LE & Lands/End Inc & SKS & Saks Inc \\
LMT & Lockheed Martin Corp & UTX & United Technologies Corp \\
S & Sears Roebuck Corp & TGT & Target Corporation \\
SGY & Stone Energy Corp & NFX & Newfield Exploration Co \\
STT & State Street Corp & MEL & Mellon Financial Corp \\
UBS & UBS AG Ord SHS & SNV & Synovus Financial Corp \\
\hline
\end{tabular}


Table 3: Descriptive statistics for CG18 (above) and TG18 (below).

\begin{tabular}{|c|c|c|c|c|c|c|c|c|c|c|}
\hline 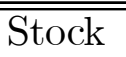 & \multicolumn{5}{|c|}{ Before decimalization } & \multicolumn{5}{|c|}{ "After decimalization } \\
\hline & $\$$ & Quotes & $\mathrm{LB}^{q}$ & Trades & $\mathrm{LB}^{t}$ & $\$$ & Quotes & $\mathrm{LB}^{q}$ & Trades & $\mathrm{LB}^{t}$ \\
\hline $\mathrm{APC}$ & 64 & 1344 & 78 & 1056 & 28 & 65 & 2233 & 70 & 1632 & 28 \\
\hline BEN & 38 & 1156 & 32 & 500 & 25 & 41 & 1577 & 42 & 709 & 25 \\
\hline $\mathrm{CI}$ & 124 & 1524 & 38 & 827 & 25 & 108 & 2252 & 45 & 1012 & 24 \\
\hline $\mathrm{CL}$ & 59 & 1366 & 80 & 856 & 35 & 56 & 2172 & 65 & 1116 & 28 \\
\hline CPQ & 21 & 1953 & 148 & 2089 & 129 & 20 & 2091 & 138 & 1825 & 59 \\
\hline DCX & 43 & 795 & 26 & 456 & 45 & 48 & 905 & 30 & 347 & 29 \\
\hline FDX & 44 & 1106 & 44 & 704 & 24 & 42 & 1661 & 35 & 835 & 26 \\
\hline GMH & 25 & 1051 & 45 & 780 & 25 & 22 & 1457 & 32 & 810 & 32 \\
\hline GT & 20 & 934 & 33 & 522 & 33 & 25 & 1524 & 39 & 663 & 30 \\
\hline GTW & 25 & 1281 & 67 & 1037 & 31 & 17 & 1630 & 60 & 1012 & 31 \\
\hline $\mathrm{H}$ & 56 & 359 & 33 & 154 & 29 & 56 & 578 & 36 & 216 & 30 \\
\hline $\mathrm{IOM}$ & 5 & 462 & 24 & 336 & 34 & 4 & 463 & 28 & 184 & 23 \\
\hline LE & 25 & 464 & 32 & 184 & 28 & 27 & 560 & 32 & 168 & 24 \\
\hline LMT & 33 & 1006 & 26 & 592 & 26 & 37 & 1403 & 32 & 798 & 28 \\
\hline $\mathrm{S}$ & 33 & 1350 & 42 & 698 & 32 & 37 & 1598 & 37 & 846 & 29 \\
\hline SGY & 57 & 506 & 41 & 202 & 29 & 54 & 612 & 40 & 237 & 26 \\
\hline $\mathrm{STT}$ & 123 & 1472 & 79 & 951 & 29 & 97 & 2414 & 54 & 1298 & 35 \\
\hline UBS & 154 & 527 & 26 & 237 & 20 & 153 & 676 & 37 & 179 & 21 \\
\hline Avg & 53 & 1036 & 50 & 677 & 35 & 51 & 1434 & 47 & 772 & 29 \\
\hline APA & 62 & 1238 & 34 & 847 & 31 & 61 & 1556 & 51 & 1120 & 31 \\
\hline $\mathrm{BSC}$ & 52 & 1463 & 29 & 815 & 34 & 51 & 2210 & 32 & 1114 & 23 \\
\hline WLP & 106 & 1111 & 49 & 716 & 22 & 96 & 1389 & 58 & 680 & 26 \\
\hline AVP & 44 & 1011 & 31 & 613 & 33 & 40 & 1427 & 42 & 828 & 30 \\
\hline $\mathrm{EMC}$ & 76 & 2738 & 135 & 2696 & 41 & 41 & 2623 & 119 & 2675 & 36 \\
\hline HDI & 42 & 1034 & 37 & 717 & 36 & 41 & 1499 & 42 & 1038 & 29 \\
\hline UPS & 59 & 961 & 28 & 550 & 55 & 57 & 1256 & 39 & 582 & 34 \\
\hline $\mathrm{F}$ & 25 & 1473 & 34 & 1100 & 137 & 28 & 1738 & 42 & 1380 & 48 \\
\hline CTB & 10 & 505 & 31 & 178 & 41 & 13 & 851 & 29 & 271 & 23 \\
\hline LXK & 45 & 1082 & 58 & 662 & 24 & 50 & 1791 & 59 & 940 & 34 \\
\hline $\mathrm{AM}$ & 11 & 479 & 23 & 227 & 37 & 13 & 633 & 29 & 239 & 24 \\
\hline DBD & 31 & 460 & 23 & 231 & 19 & 28 & 590 & 28 & 240 & 21 \\
\hline SKS & 10 & 400 & 25 & 154 & 27 & 13 & 622 & 30 & 212 & 23 \\
\hline UTX & 72 & 1393 & 50 & 847 & 28 & 76 & 2021 & 74 & 1217 & 34 \\
\hline TGT & 31 & 1379 & 35 & 1047 & 97 & 36 & 1863 & 63 & 1443 & 32 \\
\hline NFX & 41 & 446 & 26 & 214 & 19 & 36 & 630 & 27 & 277 & 18 \\
\hline MEL & 48 & 1447 & 30 & 999 & 71 & 43 & 1893 & 66 & 1363 & 37 \\
\hline SNV & 24 & 783 & 31 & 353 & 45 & 27 & 1354 & 37 & 563 & 26 \\
\hline Avg & 44 & 1078 & 39 & 720 & 44 & 42 & 1441 & 48 & 899 & 29 \\
\hline
\end{tabular}

Note: All values are based on daily averages. The Ljung-Box (LB) test is for 15 lags. Superscripts $q$ and $t$ denote the midquote and trade price data, respectively. 
Table 4: Descriptive statistics for TGDJ.

\begin{tabular}{|c|c|c|c|c|c|c|c|c|c|c|}
\hline Stock & \multicolumn{5}{|c|}{ 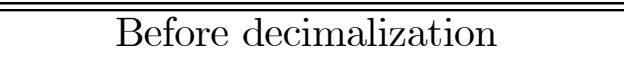 } & \multicolumn{5}{|c|}{ After decimalization } \\
\hline & $\$$ & Quotes & $\mathrm{LB}^{q}$ & Trades & $\mathrm{LB}^{t}$ & $\$$ & Quotes & $\mathrm{LB}^{q}$ & Trades & $\mathrm{LB}^{t}$ \\
\hline AA & 31 & 1466 & 28 & 1110 & 126 & 36 & 1981 & 44 & 1562 & 70 \\
\hline AIG & 95 & 2038 & 60 & 1699 & 102 & 81 & 2421 & 54 & 2191 & 60 \\
\hline AXP & 54 & 2269 & 52 & 1848 & 145 & 42 & 2706 & 95 & 2503 & 60 \\
\hline BA & 64 & 1842 & 100 & 1271 & 50 & 59 & 2531 & 72 & 1642 & 39 \\
\hline $\mathrm{C}$ & 51 & 3874 & 123 & 2768 & 372 & 48 & 3339 & 75 & 2659 & 41 \\
\hline CAT & 42 & 1175 & 28 & 804 & 68 & 44 & 1701 & 41 & 1142 & 33 \\
\hline DD & 44 & 2292 & 30 & 1376 & 152 & 43 & 2743 & 83 & 1847 & 73 \\
\hline DIS & 30 & 1651 & 34 & 1345 & 201 & 29 & 2132 & 68 & 1570 & 58 \\
\hline EK & 42 & 1369 & 39 & 872 & 87 & 43 & 1765 & 43 & 983 & 31 \\
\hline GE & 50 & 3083 & 54 & 2803 & 401 & 44 & 2497 & 161 & 2680 & 65 \\
\hline GM & 53 & 1732 & 56 & 1042 & 73 & 54 & 1982 & 106 & 1255 & 38 \\
\hline HD & 43 & 2411 & 62 & 2297 & 316 & 43 & 2520 & 135 & 2490 & 105 \\
\hline $\mathrm{HON}$ & 49 & 2064 & 29 & 1231 & 82 & 44 & 2074 & 35 & 1272 & 30 \\
\hline HWP & 34 & 2760 & 42 & 2090 & 178 & 31 & 3370 & 59 & 2479 & 80 \\
\hline IBM & 95 & 3549 & 118 & 2757 & 192 & 102 & 3764 & 114 & 3308 & 50 \\
\hline IP & 37 & 1578 & 30 & 1117 & 100 & 37 & 2110 & 43 & 1396 & 47 \\
\hline JNJ & 97 & 1966 & 59 & 1653 & 153 & 93 & 2164 & 93 & 1861 & 71 \\
\hline $\mathrm{KO}$ & 59 & 1691 & 38 & 1322 & 143 & 50 & 1679 & 79 & 1454 & 36 \\
\hline LU & 18 & 4389 & 60 & 3996 & 941 & 12 & 3138 & 115 & 3209 & 208 \\
\hline MCD & 32 & 1750 & 24 & 1105 & 177 & 28 & 2019 & 47 & 1645 & 91 \\
\hline MMM & 108 & 1416 & 96 & 1094 & 35 & 109 & 1973 & 77 & 1393 & 34 \\
\hline $\mathrm{MO}$ & 40 & 3066 & 84 & 1450 & 196 & 47 & 2437 & 144 & 1717 & 42 \\
\hline MRK & 89 & 2593 & 90 & 2149 & 183 & 76 & 1842 & 65 & 1644 & 34 \\
\hline PFE & 44 & 3195 & 46 & 2804 & 534 & 42 & 2956 & 53 & 2877 & 166 \\
\hline $\mathrm{PG}$ & 73 & 2253 & 51 & 1506 & 100 & 68 & 2380 & 53 & 1689 & 55 \\
\hline $\mathrm{SBC}$ & 52 & 2239 & 57 & 1807 & 208 & 45 & 2062 & 250 & 1942 & 45 \\
\hline $\mathrm{T}$ & 20 & 1807 & 27 & 2256 & 499 & 22 & 1741 & 157 & 1717 & 53 \\
\hline $\mathrm{VZ}$ & 54 & 2291 & 33 & 1525 & 185 & 49 & 2820 & 62 & 2098 & 60 \\
\hline WMT & 51 & 2768 & 60 & 2062 & 242 & 50 & 2021 & 123 & 1975 & 41 \\
\hline $\mathrm{XOM}$ & 87 & 2013 & 65 & 1632 & 154 & 82 & 2004 & 104 & 1754 & 43 \\
\hline Avg & 55 & 2286 & 56 & 1760 & 213 & 52 & 2362 & 88 & 1932 & 62 \\
\hline
\end{tabular}

Note: All values are based on daily averages. The Ljung-Box (LB) test is for 15 lags. Superscripts $q$ and $t$ denote the midquote and trade price data, respectively. 
Table 5: MSRV volatility estimates for CG18 (above) and TG18 (below).

\begin{tabular}{|c|c|c|c|c|c|c|c|c|}
\hline Stock & \multicolumn{4}{|c|}{ Before decimalization } & \multicolumn{4}{|c|}{ "After decimalization } \\
\hline & MSRV $^{t}$ & $\left(\%^{q}\right)$ & $\left(\%_{r v}^{t}\right)$ & $\left(\%_{r v}^{q}\right)$ & $\mathrm{MSRV}^{t}$ & $\left(\%^{q}\right)$ & $\left(\%_{r v}^{t}\right)$ & $\left(\%_{r v}^{q}\right)$ \\
\hline $\mathrm{APC}$ & $8.23 e-04$ & 3 & 25 & 44 & $\downarrow 5.38 e-04$ & 8 & 8 & 32 \\
\hline $\mathrm{BEN}$ & $5.77 e-04$ & 0 & 26 & 32 & $\downarrow 5.15 e-04$ & 2 & 27 & 14 \\
\hline $\mathrm{CI}$ & $6.12 e-04$ & 1 & 12 & 8 & $\downarrow 4.22 e-04$ & 7 & -1 & -23 \\
\hline $\mathrm{CL}$ & $4.92 e-04$ & 4 & 39 & 50 & $\downarrow 3.46 e-04$ & 4 & 4 & 24 \\
\hline $\mathrm{CPQ}$ & $1.68 e-03$ & 3 & -50 & 54 & $\downarrow 1.52 e-03$ & 3 & -16 & 48 \\
\hline DCX & $3.20 e-04$ & 6 & -120 & -10 & $\downarrow 2.07 e-04$ & 4 & -46 & -10 \\
\hline FDX & $5.61 e-04$ & 9 & 13 & 38 & $\downarrow 5.01 e-04$ & 5 & -9 & 16 \\
\hline GMH & $1.53 e-03$ & 5 & 0 & 34 & $\downarrow 9.95 e-04$ & 3 & -36 & 17 \\
\hline GT & $6.71 e-04$ & 4 & -39 & -29 & $\downarrow 5.44 e-04$ & 7 & -56 & -45 \\
\hline GTW & $2.52 e-03$ & 5 & -5 & 33 & $2.55 e-03$ & 5 & 23 & 45 \\
\hline $\mathrm{H}$ & $2.20 e-05$ & 5 & -188 & -120 & $\downarrow 1.81 e-05$ & 3 & -57 & -54 \\
\hline $\mathrm{IOM}$ & $2.37 e-03$ & 6 & -103 & -11 & $\downarrow 1.11 e-03$ & 10 & -140 & -87 \\
\hline LE & $1.15 e-03$ & -3 & 38 & 29 & $\downarrow 7.66 e-04$ & -6 & 27 & 24 \\
\hline LMT & $8.23 e-04$ & 3 & -13 & 11 & $\downarrow 6.11 e-04$ & 5 & -29 & 3 \\
\hline $\mathrm{S}$ & $6.32 e-04$ & 0 & 14 & 39 & $\downarrow 4.43 e-04$ & 2 & -1 & 7 \\
\hline SGY & $6.15 e-04$ & 4 & 35 & 42 & $6.62 e-04$ & 3 & 33 & 38 \\
\hline $\mathrm{STT}$ & $7.50 e-04$ & 3 & 19 & 52 & $\downarrow 7.17 e-04$ & 8 & -10 & 25 \\
\hline UBS & $1.04 e-04$ & 8 & 6 & 17 & $1.40 e-04$ & 5 & 14 & -9 \\
\hline Avg & $9.03 e-04$ & 4 & -16 & 17 & $\downarrow 7.00 e-04$ & 4 & -15 & 4 \\
\hline $\mathrm{APA}$ & $6.28 e-04$ & 6 & -1 & 30 & $\downarrow 4.99 e-04$ & 7 & 10 & 22 \\
\hline $\mathrm{BSC}$ & $8.08 e-04$ & 6 & -46 & 10 & $8.61 e-04$ & 5 & -18 & -13 \\
\hline WLP & $8.84 e-04$ & 4 & 21 & 39 & $\downarrow 5.78 e-04$ & 10 & 25 & 33 \\
\hline AVP & $6.37 e-04$ & 2 & -19 & 26 & $\downarrow 4.35 e-04$ & 1 & 20 & 25 \\
\hline $\mathrm{EMC}$ & $2.27 e-03$ & 2 & -20 & 31 & $3.29 e-03$ & 1 & -17 & 9 \\
\hline HDI & $1.05 e-03$ & 4 & -7 & 43 & $\downarrow 6.99 e-04$ & 6 & -19 & 25 \\
\hline UPS & $2.29 e-04$ & 1 & -78 & 17 & $\downarrow 1.44 e-04$ & 7 & -64 & -18 \\
\hline $\mathrm{F}$ & $6.75 e-04$ & 6 & -233 & 7 & $\downarrow 4.21 e-04$ & 7 & -77 & 12 \\
\hline CTB & $5.01 e-04$ & -1 & -353 & -86 & $7.49 e-04$ & 0 & -48 & -68 \\
\hline LXK & $1.85 e-03$ & 4 & 18 & 42 & $\downarrow 1.29 e-03$ & 4 & 31 & 33 \\
\hline $\mathrm{AM}$ & $1.33 e-03$ & -4 & -211 & -34 & $\downarrow 8.23 e-04$ & 0 & 11 & 9 \\
\hline DBD & $9.88 e-04$ & -3 & -1 & 13 & $\downarrow 4.89 e-04$ & -8 & 22 & -66 \\
\hline $\mathrm{SKS}$ & $1.06 e-03$ & 3 & -183 & -59 & $\downarrow 7.69 e-04$ & 15 & -56 & -12 \\
\hline UTX & $5.03 e-04$ & 5 & 1 & 40 & $\downarrow 4.56 e-04$ & 2 & 24 & 37 \\
\hline TGT & $9.51 e-04$ & 4 & -89 & 19 & $\downarrow 8.56 e-04$ & 7 & -5 & 33 \\
\hline NFX & $7.00 e-04$ & 4 & -1 & 11 & $7.23 e-04$ & 7 & 9 & 23 \\
\hline MEL & $5.97 e-04$ & 3 & -73 & 25 & $\downarrow 5.27 e-04$ & 7 & -8 & 31 \\
\hline SNV & $2.84 e-04$ & 5 & -200 & -43 & $3.15 e-04$ & 11 & -26 & -19 \\
\hline Avg & $8.86 e-04$ & 3 & -82 & 7 & $\downarrow 7.74 e-04$ & 5 & -10 & 5 \\
\hline
\end{tabular}

Note: Bolded percentages denote estimates that have become closer to MSRV ${ }^{t}$ : $\%^{q}=\frac{M S R V^{t}-M S R V^{q}}{M S R V^{t}} \times 100 ; \%_{r v}^{t}=\frac{M S R V^{t}-R V^{t}}{M S R V^{t}} \times 100 ; \%_{r v}^{q}=\frac{M S R V^{t}-R V^{q}}{M S R V^{t}} \times 100$. 
Table 6: MSRV volatility estimates for TGDJ.

\begin{tabular}{|c|c|c|c|c|c|c|c|c|}
\hline Stock & \multicolumn{4}{|c|}{ "Before decimalization } & \multicolumn{4}{|c|}{ "After decimalization } \\
\hline & $\mathrm{MSRV}^{t}$ & $\left(\%^{q}\right)$ & $\left(\%_{r v}^{t}\right)$ & $\left(\%_{r v}^{q}\right)$ & MSRV $^{t}$ & $\left(\%^{q}\right)$ & $\left(\%_{r v}^{t}\right)$ & $\left(\%_{r v}^{q}\right)$ \\
\hline $\mathrm{AA}$ & $9.04 e-04$ & 5 & -126 & 16 & $5.44 e-04$ & 3 & -37 & 30 \\
\hline $\mathrm{AIG}$ & $3.96 e-04$ & 1 & -31 & 39 & $2.92 e-04$ & 7 & -18 & 22 \\
\hline AXP & $9.20 e-04$ & 3 & -67 & 26 & $\downarrow 8.46 e-04$ & 12 & -3 & 32 \\
\hline $\mathrm{BA}$ & $6.18 e-04$ & 4 & -4 & 49 & $\downarrow 4.69 e-04$ & 3 & 3 & 35 \\
\hline $\mathrm{C}$ & $7.89 e-04$ & 5 & -137 & 31 & $\downarrow 6.69 e-04$ & 6 & -46 & -10 \\
\hline CAT & $5.36 e-04$ & 2 & -66 & 22 & $\downarrow 4.44 e-04$ & 6 & 5 & 23 \\
\hline DD & $6.15 e-04$ & 4 & -174 & 14 & $\downarrow 4.45 e-04$ & 6 & -25 & 35 \\
\hline DIS & $6.90 e-04$ & 3 & -269 & 3 & $\downarrow 6.55 e-04$ & 7 & -46 & 28 \\
\hline EK & $6.83 e-04$ & 1 & -43 & 32 & $\downarrow 5.79 e-04$ & 4 & 24 & 32 \\
\hline GE & $4.91 e-04$ & 2 & -299 & 17 & $\downarrow 4.79 e-04$ & 1 & -46 & 30 \\
\hline GM & $5.95 e-04$ & 6 & -24 & 36 & $\downarrow 3.88 e-04$ & 5 & 13 & 48 \\
\hline HD & $8.14 e-04$ & 5 & -206 & 29 & $\downarrow 7.38 e-04$ & 4 & -27 & 44 \\
\hline $\mathrm{HON}$ & $4.97 e-04$ & 3 & -82 & 0 & $5.52 e-04$ & 4 & -24 & -2 \\
\hline HWP & $1.71 e-03$ & 4 & -142 & 14 & $\downarrow 1.28 e-03$ & 1 & -71 & 18 \\
\hline IBM & $8.46 e-04$ & 4 & -64 & 31 & $\downarrow 6.69 e-04$ & 1 & -22 & 28 \\
\hline IP & $8.42 e-04$ & 4 & -69 & 22 & $\downarrow 7.09 e-04$ & 7 & -27 & 24 \\
\hline JNJ & $2.54 e-04$ & 6 & -99 & 31 & $\downarrow 1.98 e-04$ & 4 & -29 & 37 \\
\hline $\mathrm{KO}$ & $3.67 e-04$ & 4 & -111 & 28 & $4.64 e-04$ & 2 & 15 & 39 \\
\hline $\mathrm{LU}$ & $1.97 e-03$ & 6 & -991 & 3 & $2.31 e-03$ & -4 & -113 & 10 \\
\hline MCD & $4.21 e-04$ & 5 & -341 & -1 & $\downarrow 4.02 e-04$ & 5 & -99 & 18 \\
\hline MMM & $4.30 e-04$ & 5 & 17 & 47 & $4.77 e-04$ & 5 & 20 & 37 \\
\hline $\mathrm{MO}$ & $6.82 e-04$ & 4 & -111 & 37 & $\downarrow 3.86 e-04$ & 5 & 12 & 53 \\
\hline MRK & $3.60 e-04$ & 4 & -70 & 32 & $\downarrow 3.49 e-04$ & 2 & 2 & 29 \\
\hline $\mathrm{PFE}$ & $5.18 e-04$ & 5 & -467 & -13 & $\downarrow 3.76 e-04$ & 3 & -138 & 14 \\
\hline PG & $4.62 e-04$ & 9 & -71 & 24 & $\downarrow 3.85 e-04$ & 7 & -52 & 15 \\
\hline $\mathrm{SBC}$ & $5.07 e-04$ & 2 & -110 & 34 & $6.53 e-04$ & 3 & 23 & 61 \\
\hline $\mathrm{T}$ & $7.45 e-04$ & 6 & -738 & -9 & $\downarrow 6.68 e-04$ & 5 & -29 & 49 \\
\hline $\mathrm{VZ}$ & $4.83 e-04$ & 4 & -144 & 5 & $6.07 e-04$ & 8 & -30 & 13 \\
\hline WMT & $7.20 e-04$ & 4 & -122 & 29 & $\downarrow 6.40 e-04$ & 3 & 3 & 41 \\
\hline $\mathrm{XOM}$ & $2.48 e-04$ & 5 & -71 & 33 & $\downarrow 2.05 e-04$ & 10 & -2 & 35 \\
\hline Avg & $6.70 e-04$ & 4 & -174 & 22 & $\downarrow 5.96 e-04$ & 4 & -26 & 29 \\
\hline
\end{tabular}

Note: Bolded percentages denote estimates that have become closer to MSRV ${ }^{t}$ : $\%^{q}=\frac{M S R V^{t}-M S R V^{q}}{M S R V^{t}} \times 100 ; \%_{r v}^{t}=\frac{M S R V^{t}-R V^{t}}{M S R V^{t}} \times 100 ; \%_{r v}^{q}=\frac{M S R V^{t}-R V^{q}}{M S R V^{t}} \times 100$. 
Table 7: Noise variance estimates for CG18 (above) and TG18 (below).

\begin{tabular}{|c|c|c|c|c|c|c|}
\hline Stock & \multicolumn{3}{|c|}{ Before decimalization } & \multicolumn{3}{|c|}{ "After decimalization } \\
\hline & $\mathrm{RSRV}^{t}$ & $\left(\%^{q}\right)$ & $\operatorname{Cov}_{1}^{t}$ & $\mathrm{RSRV}^{t}$ & $\left(\%^{q}\right)$ & $\operatorname{Cov}_{1}^{t}$ \\
\hline $\mathrm{APC}$ & $2.91 e-07$ & 41 & $2.34 e-08$ & $1.51 e-07$ & 46 & $-5.55 e-09$ \\
\hline $\mathrm{BEN}$ & $4.24 e-07$ & 60 & $1.82 e-08$ & $\downarrow 2.65 e-07$ & 47 & $1.28 e-08$ \\
\hline CI & $3.24 e-07$ & 43 & $-7.39 e-09$ & $\downarrow 2.11 e-07$ & 46 & $-8.77 e-09$ \\
\hline $\mathrm{CL}$ & $1.76 e-07$ & 49 & $1.21 e-08$ & $\downarrow 1.49 e-07$ & 59 & $-1.57 e-08$ \\
\hline $\mathrm{CPQ}$ & $6.06 e-07$ & 67 & $-2.36 e-07$ & $\downarrow 4.83 e-07$ & 61 & $\downarrow-1.15 e-07$ \\
\hline DCX & $7.74 e-07$ & 71 & $-3.15 e-07$ & $\downarrow 4.37 e-07$ & 71 & $\downarrow-1.18 e-07$ \\
\hline FDX & $3.47 e-07$ & 55 & $-1.92 e-08$ & $\downarrow 3.28 e-07$ & 61 & $-2.73 e-08$ \\
\hline GMH & $9.85 e-07$ & 51 & $-9.52 e-08$ & $\downarrow 8.39 e-07$ & 66 & $-1.78 e-07$ \\
\hline GT & $8.93 e-07$ & 48 & $-1.69 e-07$ & $\downarrow 6.42 e-07$ & 60 & $\downarrow-1.18 e-07$ \\
\hline GTW & $1.28 e-06$ & 48 & $-3.12 e-08$ & $\downarrow 9.69 e-07$ & 55 & $-3.25 e-08$ \\
\hline $\mathrm{H}$ & $2.07 e-07$ & 67 & $-9.29 e-08$ & $\downarrow 6.60 e-08$ & 63 & $\downarrow-2.06 e-08$ \\
\hline IOM & $7.15 e-06$ & 60 & $-2.17 e-06$ & $7.28 e-06$ & 69 & $\downarrow-2.14 e-06$ \\
\hline LE & $1.93 e-06$ & 55 & $4.16 e-07$ & $\downarrow 1.66 e-06$ & 69 & $2.86 e-07$ \\
\hline LMT & $7.83 e-07$ & 53 & $-6.20 e-08$ & $\downarrow 4.94 e-07$ & 57 & $\downarrow-6.01 e-08$ \\
\hline S & $3.91 e-07$ & 64 & $-3.99 e-08$ & $\downarrow 2.64 e-07$ & 51 & $\downarrow-3.95 e-08$ \\
\hline SGY & $9.89 e-07$ & 65 & $2.04 e-07$ & $\downarrow 9.37 e-07$ & 64 & $2.05 e-07$ \\
\hline $\mathrm{STT}$ & $3.21 e-07$ & 62 & $-4.08 e-08$ & $\downarrow 3.03 e-07$ & 63 & $-5.39 e-08$ \\
\hline UBS & $2.08 e-07$ & 60 & $2.37 e-08$ & $3.35 e-07$ & 66 & $1.69 e-08$ \\
\hline Avg & $1.00 e-06$ & 57 & $-1.43 e-07$ & $8.78 e-07$ & 60 & $-1.34 e-07$ \\
\hline APA & $3.73 e-07$ & 53 & $-6.11 e-08$ & $2.02 e-07$ & 38 & $9.84 e-09$ \\
\hline $\mathrm{BSC}$ & $7.26 e-07$ & 66 & $-1.60 e-07$ & $\downarrow 4.58 e-07$ & 52 & $-1.66 e-08$ \\
\hline WLP & $4.89 e-07$ & 50 & $2.54 e-08$ & $\downarrow 3.20 e-07$ & 56 & $2.81 e-08$ \\
\hline AVP & $6.19 e-07$ & 62 & $-1.47 e-07$ & $\downarrow 2.10 e-07$ & 46 & $\downarrow-8.83 e-09$ \\
\hline EMC & $5.03 e-07$ & 43 & $-2.56 e-08$ & $7.21 e-07$ & 21 & $9.67 e-09$ \\
\hline HDI & $7.81 e-07$ & 63 & $-1.96 e-07$ & $\downarrow 4.01 e-07$ & 56 & $\downarrow-4.54 e-08$ \\
\hline UPS & $3.70 e-07$ & 73 & $-1.78 e-07$ & $\downarrow 2.04 e-07$ & 67 & $\downarrow-5.39 e-08$ \\
\hline $\mathrm{F}$ & $1.02 e-06$ & 79 & $-6.57 e-07$ & $\downarrow 2.70 e-07$ & 61 & $\downarrow-6.38 e-08$ \\
\hline CTB & $6.36 e-06$ & 85 & $-4.08 e-06$ & $\downarrow 2.04 e-06$ & 64 & $\downarrow-2.93 e-07$ \\
\hline LXK & $1.15 e-06$ & 57 & $-1.94 e-08$ & $\downarrow 4.72 e-07$ & 49 & $7.17 e-08$ \\
\hline $\mathrm{AM}$ & $9.14 e-06$ & 80 & $-5.34 e-06$ & $\downarrow 1.53 e-06$ & 61 & $\downarrow-1.80 e-07$ \\
\hline DBD & $2.16 e-06$ & 57 & $-6.20 e-08$ & $\downarrow 7.98 e-07$ & 14 & $1.09 e-07$ \\
\hline SKS & $9.78 e-06$ & 78 & $-4.07 e-06$ & $\downarrow 2.84 e-06$ & 76 & $\downarrow-3.24 e-07$ \\
\hline UTX & $2.93 e-07$ & 63 & $-4.25 e-08$ & $\downarrow 1.43 e-07$ & 50 & $1.31 e-08$ \\
\hline TGT & $8.59 e-07$ & 68 & $-4.58 e-07$ & $\downarrow 3.11 e-07$ & 51 & $\downarrow-3.12 e-08$ \\
\hline NFX & $1.66 e-06$ & 58 & $6.92 e-08$ & $\downarrow 1.18 e-06$ & 63 & $3.56 e-08$ \\
\hline MEL & $5.16 e-07$ & 70 & $-2.28 e-07$ & $\downarrow 2.08 e-07$ & 54 & $\downarrow-2.71 e-08$ \\
\hline SNV & $1.21 e-06$ & 78 & $-6.47 e-07$ & $\downarrow 3.52 e-07$ & 61 & $\downarrow-4.79 e-08$ \\
\hline Avg & $2.11 e-06$ & 66 & $-9.04 e-07$ & $\downarrow 7.03 e-07$ & 52 & $\downarrow-4.53 e-08$ \\
\hline
\end{tabular}

Note: Bolded percentages denote estimates that have become closer to RSRV ${ }^{t}$ : $\%^{q}=\frac{R S R V^{t}-R S R V^{q}}{R S R V^{t}} \times 100$. 
Table 8: Noise variance estimates for TGDJ.

\begin{tabular}{|c|c|c|c|c|c|c|}
\hline Stock & \multicolumn{3}{|c|}{ Before decimalization } & \multicolumn{3}{|c|}{ After decimalization } \\
\hline & $\mathrm{RSRV}^{t}$ & $\left(\%^{q}\right)$ & $\operatorname{Cov}_{1}^{t}$ & $\mathrm{RSRV}^{t}$ & $\left(\%^{q}\right)$ & $\operatorname{Cov}_{1}^{t}$ \\
\hline AA & $9.19 e-07$ & 71 & $-5.61 e-07$ & $2.39 e-07$ & 60 & $\downarrow-7.18 e-08$ \\
\hline $\mathrm{AIG}$ & $1.53 e-07$ & 61 & $-6.05 e-08$ & $7.86 e-08$ & 40 & $\downarrow-1.49 e-08$ \\
\hline AXP & $4.15 e-07$ & 64 & $-2.03 e-07$ & $\downarrow 1.74 e-07$ & 39 & $\downarrow-2.70 e-08$ \\
\hline $\mathrm{BA}$ & $2.54 e-07$ & 66 & $-7.14 e-08$ & $\downarrow 1.39 e-07$ & 56 & $\downarrow-1.27 e-08$ \\
\hline $\mathrm{C}$ & $3.38 e-07$ & 79 & $-2.30 e-07$ & $\downarrow 1.84 e-07$ & 40 & $\downarrow-7.51 e-10$ \\
\hline CAT & $5.51 e-07$ & 68 & $-2.57 e-07$ & $\downarrow 1.85 e-07$ & 46 & $\downarrow-2.34 e-08$ \\
\hline DD & $6.11 e-07$ & 81 & $-3.72 e-07$ & $\downarrow 1.51 e-07$ & 65 & $\downarrow-4.37 e-08$ \\
\hline DIS & $9.46 e-07$ & 79 & $-6.84 e-07$ & $\downarrow 3.04 e-07$ & 63 & $\downarrow-7.13 e-08$ \\
\hline EK & $5.59 e-07$ & 70 & $-2.89 e-07$ & $\downarrow 2.23 e-07$ & 50 & $\downarrow-1.01 e-08$ \\
\hline GE & $3.50 e-07$ & 81 & $-2.53 e-07$ & $.31 e-07$ & 49 & $\downarrow-1.78 e-08$ \\
\hline GM & $3.55 e-07$ & 69 & $-1.43 e-07$ & $\downarrow 1.34 e-07$ & 62 & $\downarrow-1.29 e-08$ \\
\hline HD & $5.41 e-07$ & 78 & $-3.81 e-07$ & $\downarrow 1.89 e-07$ & 56 & $\downarrow-5.61 e-08$ \\
\hline $\mathrm{HON}$ & $3.67 e-07$ & 67 & $-1.56 e-07$ & $\downarrow 2.68 e-07$ & 50 & $\downarrow-2.13 e-08$ \\
\hline HWP & $9.87 e-07$ & 73 & $-5.16 e-07$ & $\downarrow 4.42 e-07$ & 65 & $\downarrow-9.35 e-08$ \\
\hline IBM & $2.52 e-07$ & 67 & $-1.16 e-07$ & $24 e-07$ & 49 & $\downarrow-1.08 e-08$ \\
\hline IP & $6.36 e-07$ & 67 & $-3.28 e-07$ & $\downarrow 3.23 e-07$ & 61 & $\downarrow-6.13 e-08$ \\
\hline JNJ & $1.53 e-07$ & 71 & $-8.29 e-08$ & $\downarrow 6.85 e-08$ & 58 & $\downarrow-1.99 e-08$ \\
\hline $\mathrm{KO}$ & $2.93 e-07$ & 73 & $-1.76 e-07$ & $\downarrow 1.36 e-07$ & 38 & $\downarrow-1.12 e-08$ \\
\hline $\mathrm{LU}$ & $2.69 e-06$ & 92 & $-2.59 e-06$ & $\downarrow 7.68 e-07$ & 57 & $\downarrow-2.96 e-07$ \\
\hline MCD & $8.41 e-07$ & 86 & $-6.31 e-07$ & $\downarrow 2.43 e-07$ & 67 & $\downarrow-9.29 e-08$ \\
\hline MMM & $1.64 e-07$ & 51 & $-1.67 e-08$ & $\downarrow 1.38 e-07$ & 45 & $1.38 e-08$ \\
\hline $\mathrm{MO}$ & $4.96 e-07$ & 86 & $-3.34 e-07$ & $\downarrow 9.92 e-08$ & 63 & $\downarrow-1.07 e-08$ \\
\hline MRK & $1.42 e-07$ & 67 & $-7.11 e-08$ & $\downarrow 1.04 e-07$ & 35 & $\downarrow-3.60 e-09$ \\
\hline PFE & $5.24 e-07$ & 83 & $-4.43 e-07$ & $\downarrow 1.55 e-07$ & 65 & $\downarrow-6.20 e-08$ \\
\hline PG & $2.62 e-07$ & 70 & $-1.14 e-07$ & $\downarrow 1.73 e-07$ & 60 & $\downarrow-3.89 e-08$ \\
\hline $\mathrm{SBC}$ & $2.94 e-07$ & 74 & $-1.87 e-07$ & $\downarrow 1.29 e-07$ & 53 & $\downarrow-2.93 e-09$ \\
\hline $\mathrm{T}$ & $1.38 e-06$ & 84 & $-1.27 e-06$ & $\downarrow 2.52 e-07$ & 61 & $\downarrow-5.86 e-08$ \\
\hline $\mathrm{VZ}$ & $3.86 e-07$ & 74 & $-2.44 e-07$ & $\downarrow 1.88 e-07$ & 50 & $\downarrow-4.14 e-08$ \\
\hline WMT & $3.88 e-07$ & 76 & $-2.51 e-07$ & $\downarrow 1.57 e-07$ & 41 & $\downarrow-8.96 e-09$ \\
\hline $\mathrm{XOM}$ & $1.30 e-07$ & 68 & $-7.25 e-08$ & $\downarrow 5.96 e-08$ & 44 & $\downarrow-6.68 e-09$ \\
\hline Avg & $5.46 e-07$ & 73 & $-3.70 e-07$ & $1.99 e-07$ & 53 & $\downarrow-3.96 e-08$ \\
\hline
\end{tabular}

Note: Bolded percentages denote estimates that have become closer to RSRV ${ }^{t}$ : $\%^{q}=\frac{R S R V^{t}-R S R V^{q}}{R S R V^{t}} \times 100$. 
Table 9: General test statistics.

\begin{tabular}{|c|c|c|c|c|c|}
\hline & Mean before & Mean after & Mean $(\Delta)$ & Paired $t$ & Wilcoxon \\
\hline & \multicolumn{5}{|c|}{ Control Group 18} \\
\hline Abs spread & 0.11 & 0.10 & 0.01 & $\begin{array}{c}1.12 \\
(0.2771)\end{array}$ & $\begin{array}{c}121 \\
(0.1297)\end{array}$ \\
\hline Rel spread & 0.28 & 0.27 & 0.01 & $\begin{array}{c}0.73 \\
(0.478)\end{array}$ & $\begin{array}{c}118 \\
(0.1674)\end{array}$ \\
\hline Nr quotes & 1036 & 1434 & -397 & $\begin{array}{c}-\mathbf{5 . 7 2}^{*} \\
(2.52 e-05)\end{array}$ & $\begin{array}{c}\mathbf{0}^{*} \\
(7.63 e-06)\end{array}$ \\
\hline Nr trades & 677 & 772 & -95 & $\begin{array}{l}-2.05 \\
(0.0556)\end{array}$ & $\begin{array}{c}42 \\
(0.0599)\end{array}$ \\
\hline Depth & 70252 & 78945 & -8693 & $\begin{array}{l}-1.28 \\
(0.2166)\end{array}$ & $\begin{array}{c}46 \\
(0.0898)\end{array}$ \\
\hline Volume & 1697999 & 1554826 & 143173 & $\begin{array}{c}0.97 \\
(0.3436)\end{array}$ & $\begin{array}{c}90 \\
(0.865)\end{array}$ \\
\hline $\mathrm{LB}(15)^{t}$ & 35 & 29 & 5 & $\begin{array}{c}1.34 \\
(0.1983)\end{array}$ & $\begin{array}{c}113 \\
(0.2462)\end{array}$ \\
\hline \multirow[t]{2}{*}{$\operatorname{LB}(15)^{q}$} & 50 & 47 & 2 & $\begin{array}{l}1.01 \\
(0.3247)\end{array}$ & $\begin{array}{c}105 \\
(0.4171)\end{array}$ \\
\hline & \multicolumn{5}{|c|}{ Test Group 18} \\
\hline Abs spread & 0.13 & 0.08 & 0.05 & $\begin{array}{c}\mathbf{1 2 . 8 5}^{*} \\
(3.52 e-10)\end{array}$ & $\begin{array}{c}\mathbf{1 7 1}^{\mathbf{*}} \\
(7.63 e-06)\end{array}$ \\
\hline Rel spread & 0.41 & 0.25 & 0.16 & $\begin{array}{c}\mathbf{5 . 0 9}^{*} \\
(9.16 e-05)\end{array}$ & $\begin{array}{c}\mathbf{1 7 1}^{*} \\
(7.63 e-06)\end{array}$ \\
\hline Nr quotes & 1078 & 1441 & -363 & $\begin{array}{c}-\mathbf{7 . 0 3}^{*} \\
(2.03 e-06)\end{array}$ & $\begin{array}{c}\mathbf{1}^{*} \\
(1.53 e-05)\end{array}$ \\
\hline $\mathrm{Nr}$ trades & 720 & 899 & -179 & $\begin{array}{c}-\mathbf{5 . 0 4}^{*} \\
(9.99 e-05)\end{array}$ & $\begin{array}{c}\mathbf{8}^{*} \\
(0.0002)\end{array}$ \\
\hline Depth & 127961 & 76643 & 51318 & $\begin{array}{c}\mathbf{2 . 5 9} \\
(0.0190)\end{array}$ & $\begin{array}{l}\mathbf{1 5 9}^{*} \\
(0.0005)\end{array}$ \\
\hline Volume & 1914090 & 2251013 & -336922 & $\begin{array}{l}-1.02 \\
(0.3214)\end{array}$ & $\begin{array}{c}70 \\
(0.5226)\end{array}$ \\
\hline $\mathrm{LB}(15)^{t}$ & 44 & 29 & 15 & $\begin{array}{c}2.50 \\
(0.0228)\end{array}$ & $\begin{array}{l}\mathbf{1 4 4}^{*} \\
(0.0090)\end{array}$ \\
\hline \multirow[t]{2}{*}{$\mathrm{LB}(15)^{q}$} & 39 & 48 & -9 & $\frac{-\mathbf{3 . 2 1}^{*}}{(0.0051)}$ & $\begin{array}{c}1 \mathbf{7}^{*} \\
(0.0016)\end{array}$ \\
\hline & \multicolumn{5}{|c|}{ Test Group Dow Jones } \\
\hline Abs spread & 0.11 & 0.06 & 0.05 & $\begin{array}{c}\mathbf{3 0 . 1 2}^{*} \\
(<2.2 e-16)\end{array}$ & $\begin{array}{c}\mathbf{4 6 5}^{*} \\
(1.86 e-09)\end{array}$ \\
\hline Rel spread & 0.22 & 0.13 & 0.09 & $\begin{array}{c}\mathbf{1 0 . 3 1}^{*} \\
(3.27 e-11)\end{array}$ & $\begin{array}{c}\mathbf{4 6 5}^{*} \\
(1.86 e-09)\end{array}$ \\
\hline Nr quotes & 2286 & 2362 & -76 & $\begin{array}{l}-0.84 \\
(0.4057)\end{array}$ & $\begin{array}{c}184 \\
(0.3285)\end{array}$ \\
\hline $\mathrm{Nr}$ trades & 1760 & 1932 & -172 & $\frac{-\mathbf{2 . 8 1}}{(0.0087)}$ & $\begin{array}{c}\mathbf{9 3}^{*} \\
(0.0032)\end{array}$ \\
\hline Depth & 632513 & 191075 & 441438 & $\begin{array}{l}\mathbf{3 . 0 2}^{*} \\
(0.0052)\end{array}$ & $\begin{array}{c}\mathbf{4 6 4}^{*} \\
(3.73 e-09)\end{array}$ \\
\hline Volume & 5942661 & 5812197 & 130463 & $\begin{array}{c}0.41 \\
(0.6837)\end{array}$ & $\begin{array}{l}252 \\
(0.7)\end{array}$ \\
\hline $\mathrm{LB}(15)^{t}$ & 213 & 62 & 151 & $\begin{array}{c}\mathbf{5 . 3 3}^{*} \\
(1.01 e-05)\end{array}$ & $\begin{array}{c}\mathbf{4 6 5}^{*} \\
(1.86 e-09)\end{array}$ \\
\hline $\mathrm{LB}(15)^{q}$ & 56 & 88 & -32 & $\begin{array}{c}-\mathbf{3 . 6 4}^{*} \\
(0.0011)\end{array}$ & $\begin{array}{c}\mathbf{6 5}^{*} \\
(0.0003) \\
\end{array}$ \\
\hline
\end{tabular}

Note: Absolute spread (Abs spread) denotes the absolute difference between the ask and bid quotes, relative spread (Rel spread) denotes the absolute spread standardized by the corresponding midquote, Depth denotes the sum of displayed number of quotes at ask and bid, and Volume denotes the number of traded stocks $(\times 100)$. Bolded significant at 5\% level, ${ }^{*}$ denotes $1 \%$ ( $p$-values in parentheses). 
Table 10: Test statistics for noise variance, volatility, and SNR.

\begin{tabular}{|c|c|c|c|c|c|c|}
\hline \multirow[b]{3}{*}{$\left|\operatorname{Cov}_{1}\right|$} & Mean $(\Delta)$ & Paired $t$ & $\overline{\text { Wilcoxon }}$ & $\overline{\operatorname{Mean}(\Delta)}$ & Paired $t$ & Wilcoxon \\
\hline & \multicolumn{3}{|c|}{ Control Group 18 (trade prices) } & \multicolumn{3}{|c|}{ Control Group 18 (midquotes) } \\
\hline & $3.24 e-08$ & $\begin{array}{l}\mathbf{2 . 3 0} \\
(0.0342)\end{array}$ & $\begin{array}{c}136 \\
(0.0269)\end{array}$ & $2.53 e-08$ & $\begin{array}{l}3.18^{*} \\
(0.0055)\end{array}$ & $\begin{array}{l}\mathbf{1 5 4}^{*} \\
(0.0016)\end{array}$ \\
\hline $\mathrm{RSRV}$ & $1.26 e-07$ & $\begin{array}{l}3.98^{*} \\
(0.0010)\end{array}$ & $\begin{array}{l}15 \mathbf{6}^{*} \\
(0.0010)\end{array}$ & $1.18 e-07$ & $\begin{array}{l}3.24^{*} \\
(0.0048)\end{array}$ & $\begin{array}{l}\mathbf{1 6 3}^{*} \\
(0.0002)\end{array}$ \\
\hline TSRV & $1.80 e-04$ & $\begin{array}{c}\mathbf{2 . 6 5} \\
(0.0170)\end{array}$ & $\begin{array}{l}\mathbf{1 6 1}^{*} \\
(0.0003)\end{array}$ & $1.68 e-04$ & $\begin{array}{c}\mathbf{2 . 8 1} \\
(0.0120)\end{array}$ & $\begin{array}{l}\mathbf{1 5 5 ^ { * }} \\
(0.0013)\end{array}$ \\
\hline GTSRV & $1.67 e-04$ & $\begin{array}{c}\mathbf{2 . 7 6} \\
(0.0134)\end{array}$ & $\begin{array}{l}160^{*} \\
(0.0004)\end{array}$ & $1.76 e-04$ & $\begin{array}{c}\mathbf{2 . 8 6} \\
(0.0109)\end{array}$ & $\begin{array}{l}158^{*} \\
(0.0007)\end{array}$ \\
\hline MSRV & $2.03 e-04$ & $\begin{array}{c}2.83 \\
(0.0116)\end{array}$ & $\begin{array}{l}159^{*} \\
(0.0005)\end{array}$ & $2.02 e-04$ & $\begin{array}{c}2.89 \\
(0.0101)\end{array}$ & $\begin{array}{l}160^{*} \\
(0.0004)\end{array}$ \\
\hline $\mathrm{SNR}_{1}$ & -149 & $\begin{array}{l}-1.94 \\
(0.0686)\end{array}$ & $\begin{array}{c}44 \\
(0.0737)\end{array}$ & -430 & $\frac{-\mathbf{3 . 1 8}^{*}}{(0.0054)}$ & $\begin{array}{c}\mathbf{2 3}^{*} \\
(0.0047)\end{array}$ \\
\hline $\mathrm{SNR}_{2}$ & -150 & $\begin{array}{l}-1.97 \\
(0.0658)\end{array}$ & $\begin{array}{c}45 \\
(0.0814)\end{array}$ & -431 & $\frac{-\mathbf{3 . 1 9}^{*}}{(0.0053)}$ & $\begin{array}{c}\mathbf{2 2}^{*} \\
(0.0041)\end{array}$ \\
\hline $\mathrm{SNR}_{3}$ & -102 & $\begin{array}{l}-1.31 \\
(0.2078)\end{array}$ & $\begin{array}{c}60 \\
(0.2837)\end{array}$ & -293 & $\begin{array}{l}-\mathbf{2 . 1 4} \\
(0.0467)\end{array}$ & $\begin{array}{c}\mathbf{3 1} \\
(0.0159)\end{array}$ \\
\hline \multirow[t]{2}{*}{$\mathrm{SNR}_{3 b}$} & -193624 & $\begin{array}{l}-0.81 \\
(0.4265)\end{array}$ & $\begin{array}{c}88 \\
(0.9323)\end{array}$ & $\mathrm{N} / \mathrm{A}$ & $\mathrm{N} / \mathrm{A}$ & $\mathrm{N} / \mathrm{A}$ \\
\hline & \multicolumn{3}{|c|}{ Test Group 18 (trade prices) } & \multicolumn{3}{|c|}{ Test Group 18 (midquotes) } \\
\hline$\left|\operatorname{Cov}_{1}\right|$ & $8.36 e-07$ & $\begin{array}{c}\mathbf{2 . 3 1} \\
(0.0340)\end{array}$ & $\begin{array}{c}\mathbf{1 6 9}^{*} \\
(2.29 e-05)\end{array}$ & $3.91 e-08$ & $\begin{array}{c}\mathbf{2 . 5 9} \\
(0.0192)\end{array}$ & $\begin{array}{c}\mathbf{1 5 4} \\
(0.0016)\end{array}$ \\
\hline RSRV & $1.41 e-06$ & $\begin{array}{c}\mathbf{2 . 5 4} \\
(0.0211)\end{array}$ & $\begin{array}{c}166^{*} \\
(7.63 e-05)\end{array}$ & $2.36 e-07$ & $\begin{array}{l}\mathbf{2 . 3 8} \\
(0.0295)\end{array}$ & $\begin{array}{l}\mathbf{1 5 5}^{*} \\
(0.0013)\end{array}$ \\
\hline TSRV & $9.68 e-05$ & $\begin{array}{l}1.19 \\
(0.2522)\end{array}$ & $\begin{array}{c}\mathbf{1 3 4} \\
(0.0342)\end{array}$ & $9.24 e-05$ & $\begin{array}{l}1.00 \\
(0.3275)\end{array}$ & $\begin{array}{c}133 \\
(0.0385)\end{array}$ \\
\hline GTSRV & $8.07 e-05$ & $\begin{array}{l}1.02 \\
(0.3213)\end{array}$ & $\begin{array}{c}128 \\
(0.0665)\end{array}$ & $1.09 e-04$ & $\begin{array}{c}1.14 \\
(0.2693)\end{array}$ & $\begin{array}{c}135 \\
(0.0304)\end{array}$ \\
\hline MSRV & $1.13 e-04$ & $\begin{array}{l}1.34 \\
(0.1986)\end{array}$ & $\begin{array}{c}136 \\
(0.0269)\end{array}$ & $1.23 e-04$ & $\begin{array}{l}1.44 \\
(0.1675)\end{array}$ & $\begin{array}{c}135 \\
(0.0304)\end{array}$ \\
\hline $\mathrm{SNR}_{1}$ & -694 & $\begin{array}{c}-\mathbf{5 . 6 2}^{*} \\
(3.05 e-05)\end{array}$ & $\begin{array}{c}\mathbf{0}^{*} \\
(7.63 e-06)\end{array}$ & -868 & $\begin{array}{c}\mathbf{- 5 . 3 7} \\
(5.07 e-05)\end{array}$ & $\begin{array}{c}\mathbf{4}^{*} \\
(5.34 e-05)\end{array}$ \\
\hline $\mathrm{SNR}_{2}$ & -689 & $\begin{array}{c}-\mathbf{5 . 6 3}^{*} \\
(3.02 e-05)\end{array}$ & $\begin{array}{c}\mathbf{0}^{*} \\
(7.63 e-06)\end{array}$ & -869 & $\begin{array}{c}-\mathbf{5 . 3 8} \\
(4.96 e-05)\end{array}$ & $\begin{array}{c}\mathbf{3}^{*} \\
(3.82 e-05)\end{array}$ \\
\hline $\mathrm{SNR}_{3}$ & -696 & $\begin{array}{c}-\mathbf{5 . 7 7 ^ { * }} \\
(2.27 e-05)\end{array}$ & $\begin{array}{c}\mathbf{0}^{*} \\
(7.63 e-06)\end{array}$ & -813 & $\frac{\mathbf{- 5 . 1 1}}{(8.76 e-05)}$ & $\begin{array}{c}\mathbf{4}^{*} \\
(5.34 e-05)\end{array}$ \\
\hline \multirow[t]{2}{*}{$\mathrm{SNR}_{3 b}$} & -133474 & $\begin{array}{l}-1.09 \\
(0.2924)\end{array}$ & $\begin{array}{c}48 \\
(0.1084)\end{array}$ & $\mathrm{N} / \mathrm{A}$ & $\mathrm{N} / \mathrm{A}$ & $\mathrm{N} / \mathrm{A}$ \\
\hline & \multicolumn{3}{|c|}{ Test Group DJ (trade prices) } & \multicolumn{3}{|c|}{ Test Group DJ (midquotes) } \\
\hline$\left|\operatorname{Cov}_{1}\right|$ & $3.26 e-07$ & $\begin{array}{l}\mathbf{4 . 0 6}{ }^{*} \\
(0.0003)\end{array}$ & $\begin{array}{c}\mathbf{4 6 5}^{*} \\
(1.86 e-09)\end{array}$ & $-2.72 e-09$ & $\begin{array}{l}-0.70 \\
(0.4921)\end{array}$ & $\begin{array}{c}250 \\
(0.7303)\end{array}$ \\
\hline RSRV & $3.47 e-07$ & $\begin{array}{c}\mathbf{4 . 9 7}^{*} \\
(2.76 e-05)\end{array}$ & $\begin{array}{c}\mathbf{4 6 5}^{*} \\
(1.86 e-09)\end{array}$ & $3.13 e-08$ & $\begin{array}{l}3.24^{*} \\
(0.0030)\end{array}$ & $\begin{array}{c}\mathbf{3 8 9}^{*} \\
(0.0008)\end{array}$ \\
\hline TSRV & $5.70 e-05$ & $\begin{array}{c}1.96 \\
(0.0601)\end{array}$ & $\begin{array}{c}\mathbf{3 4 7} \\
(0.0175)\end{array}$ & $5.40 e-05$ & $\begin{array}{c}1.51 \\
(0.1416)\end{array}$ & $\begin{array}{c}360^{*} \\
(0.0076)\end{array}$ \\
\hline GTSRV & $5.40 e-05$ & $\begin{array}{c}1.92 \\
(0.0651)\end{array}$ & $\begin{array}{c}\mathbf{3 4 3} \\
(0.0221)\end{array}$ & $5.64 e-05$ & $\begin{array}{c}1.55 \\
(0.1309)\end{array}$ & $\begin{array}{c}\mathbf{3 6 0}^{*} \\
(0.0076)\end{array}$ \\
\hline MSRV & $7.45 e-05$ & $\begin{array}{c}2.73 \\
(0.0105)\end{array}$ & $\begin{array}{c}368^{*} \\
(0.0043)\end{array}$ & $6.68 e-05$ & $\begin{array}{c}\mathbf{2 . 1 8} \\
(0.0375)\end{array}$ & $\begin{array}{l}378^{*} \\
(0.0020)\end{array}$ \\
\hline $\mathrm{SNR}_{1}$ & -1657 & $\frac{-\mathbf{1 3 . 9 3}^{*}}{(2.25 e-14)}$ & $\begin{array}{c}\mathbf{0}^{*} \\
(1.86 e-09)\end{array}$ & -1033 & $\frac{-\mathbf{3 . 4 4}}{(0.0018)}$ & $\begin{array}{c}79^{*} \\
(0.0010)\end{array}$ \\
\hline $\mathrm{SNR}_{2}$ & -1637 & $\frac{-13.92^{*}}{(2.29 e-14)}$ & $\begin{array}{c}\mathbf{0}^{*} \\
(1.86 e-09)\end{array}$ & -1033 & $\frac{-\mathbf{3 . 4 4}}{(0.0018)}$ & $\begin{array}{c}\mathbf{7 9}^{*} \\
(0.0010)\end{array}$ \\
\hline $\mathrm{SNR}_{3}$ & -1591 & $\frac{-13.31^{*}}{(7.09 e-14)}$ & $\begin{array}{c}\mathbf{0}^{*} \\
(1.86 e-09)\end{array}$ & -980 & $\frac{-\mathbf{3 . 2 6}^{*}}{(0.0029)}$ & $\begin{array}{c}\mathbf{8 1}^{*} \\
(0.0012)\end{array}$ \\
\hline $\mathrm{SNR}_{3 b}$ & -45476 & $\begin{array}{l}-\mathbf{2 . 3 0} \\
(0.0288)\end{array}$ & $\begin{array}{c}\mathbf{5 2}^{*} \\
(7.06 e-05)\end{array}$ & $\mathrm{N} / \mathrm{A}$ & $\mathrm{N} / \mathrm{A}$ & $\mathrm{N} / \mathrm{A}$ \\
\hline
\end{tabular}

Note: Bolded significant at 5\% level, ${ }^{*}$ denotes 1\% (p-values in parentheses). 
Table 11: Mixed-effects panel regressions using trade price data.

\begin{tabular}{|c|c|c|c|c|c|c|}
\hline \multirow[b]{4}{*}{ Intercept } & \multicolumn{6}{|c|}{ Control Group $18(18 \times 98=1764$ observations $)$} \\
\hline & \multicolumn{2}{|c|}{ Volatility } & \multicolumn{2}{|c|}{ Noise variance } & \multicolumn{2}{|c|}{$\mathrm{SNR}_{3}$} \\
\hline & Fixed & Random & Fixed & Random & Fixed & Random \\
\hline & $\begin{array}{c}-\mathbf{7 . 5 8} \\
{[-30.61]}\end{array}$ & 1.04 & $\begin{array}{c}-\mathbf{1 4 . 5 2} \\
{[-60.11]}\end{array}$ & 1.02 & $\begin{array}{l}\mathbf{6 . 8 9}^{*} \\
{[96.44]}\end{array}$ & 0.30 \\
\hline Nr trades & $\begin{array}{l}\mathbf{1 . 4 7}^{*} \\
{[17.79]}\end{array}$ & 0.25 & $\begin{array}{l}0.14 \\
{[1.74]}\end{array}$ & 0.30 & $\begin{array}{l}1.28^{*} \\
{[22.09]}\end{array}$ & 0.20 \\
\hline $\mathrm{LB}(15)$ & $\frac{-0.12}{[-1.44]}$ & 0.32 & $\begin{array}{l}-0.02 \\
{[-0.75]}\end{array}$ & 0.07 & $\begin{array}{l}-0.09 \\
{[-1.17]}\end{array}$ & 0.33 \\
\hline \multirow[t]{4}{*}{ Dummy } & $\begin{array}{c}-\mathbf{0 . 3 9}^{*} \\
{[-4.26]}\end{array}$ & 0.36 & $\begin{array}{c}-\mathbf{0 . 2 6}^{*} \\
{[-3.58]}\end{array}$ & 0.30 & $\begin{array}{l}-0.10 \\
{[-1.91]}\end{array}$ & 0.22 \\
\hline & \multicolumn{6}{|c|}{ Test Group $18(18 \times 98=1764$ observations $)$} \\
\hline & \multicolumn{2}{|c|}{ Volatility } & \multicolumn{2}{|c|}{ Noise variance } & \multicolumn{2}{|c|}{$\mathrm{SNR}_{3}$} \\
\hline & Fixed & Random & Fixed & Random & Fixed & Random \\
\hline Intercept & $\begin{array}{c}-\mathbf{7 . 0 6}^{*} \\
{[-25.29]}\end{array}$ & 1.17 & $\begin{array}{c}-\mathbf{1 3 . 8 0}^{*} \\
{[-51.18]}\end{array}$ & 1.14 & $\begin{array}{l}\mathbf{6 . 7 2}^{*} \\
{[78.16]}\end{array}$ & 0.35 \\
\hline Nr trades & $\begin{array}{l}\mathbf{1 . 7 3}^{*} \\
{[12.22]}\end{array}$ & 0.55 & $\begin{array}{c}\mathbf{0 . 2 5 ^ { * }} \\
{[4.03]}\end{array}$ & 0.20 & $\begin{array}{l}\mathbf{1 . 4 9}^{*} \\
{[17.04]}\end{array}$ & 0.33 \\
\hline $\mathrm{LB}(15)$ & $\begin{array}{c}-0.27^{*} \\
{[-3.96]}\end{array}$ & 0.26 & $\begin{array}{l}-0.03 \\
{[-1.11]}\end{array}$ & 0.09 & $\begin{array}{c}-\mathbf{0 . 2 4} \\
{[-3.32]}\end{array}$ & 0.30 \\
\hline \multirow[t]{4}{*}{ Dummy } & $\begin{array}{c}-0.77^{*} \\
{[-6.97]}\end{array}$ & 0.44 & $\begin{array}{c}-\mathbf{0 . 9 4}^{*} \\
{[-8.36]}\end{array}$ & 0.47 & $\mathbf{0 . 1 6}^{*}$ & 0.21 \\
\hline & \multicolumn{6}{|c|}{ Test Group Dow Jones $(30 \times 98=2940$ observations $)$} \\
\hline & \multicolumn{2}{|c|}{ Volatility } & \multicolumn{2}{|c|}{ Noise variance } & \multicolumn{2}{|c|}{$\mathrm{SNR}_{3}$} \\
\hline & Fixed & Random & Fixed & Random & Fixed & Random \\
\hline Intercept & $\begin{array}{c}-\mathbf{7 . 1 2}{ }^{*} \\
{[-60.12]}\end{array}$ & 0.63 & $\begin{array}{c}\mathbf{- 1 4 . 6 9} \\
{[-129.44]}\end{array}$ & 0.61 & $\begin{array}{l}7.58^{*} \\
{[130.05]}\end{array}$ & 0.30 \\
\hline Nr trades & $\begin{array}{l}\mathbf{2 . 0 2} \\
{[22.96]}\end{array}$ & 0.35 & $\begin{array}{c}\mathbf{0 . 4 7 ^ { * }} \\
{[5.84]}\end{array}$ & 0.39 & $\begin{array}{l}\mathbf{1 . 5 4}^{*} \\
{[20.33]}\end{array}$ & 0.33 \\
\hline $\mathrm{LB}(15)$ & $\begin{array}{c}-\mathbf{0 . 4 5} \\
{[-9.48]}\end{array}$ & 0.23 & $\begin{array}{l}-\mathbf{0 . 0 5} \\
{[-2.04]}\end{array}$ & 0.11 & $\frac{\mathbf{- 0 . 4 1}}{[-10.45]}$ & 0.20 \\
\hline Dummy & $\frac{-\mathbf{0 . 9 6}^{*}}{[-11.16]}$ & 0.43 & $\begin{array}{c}-\mathbf{1 . 0 6} \\
{[-16.25]}\end{array}$ & 0.34 & $\begin{array}{l}0.08 \\
{[1.80]}\end{array}$ & 0.21 \\
\hline
\end{tabular}

Note: Bolded significant at 5\% level, ${ }^{*}$ denotes $1 \%$ ( $t$-statistics in brackets). The random part denotes the variable's standard deviation (covariances not reported). 
Table 12: Mixed-effects panel regressions using midquote data.

\begin{tabular}{|c|c|c|c|c|c|c|}
\hline \multirow[b]{4}{*}{ Intercept } & \multicolumn{6}{|c|}{ Control Group $18(18 \times 98=1764$ observations $)$} \\
\hline & \multicolumn{2}{|c|}{ Volatility } & \multicolumn{2}{|c|}{ Noise variance } & \multicolumn{2}{|c|}{$\mathrm{SNR}_{3}$} \\
\hline & Fixed & Random & Fixed & Random & Fixed & Random \\
\hline & $\begin{array}{c}\mathbf{- 7 . 4 5} \\
{[-31.60]}\end{array}$ & 0.99 & $\begin{array}{c}\mathbf{- 1 5 . 2 3} \\
{[-70.43]}\end{array}$ & 0.91 & $\begin{array}{l}\mathbf{7 . 7 6}^{*} \\
{[93.93]}\end{array}$ & 0.34 \\
\hline Nr quotes & $\begin{array}{c}\mathbf{1 . 0 8}^{*} \\
{[8.45]}\end{array}$ & 0.46 & $\begin{array}{l}-0.06 \\
{[-0.53]}\end{array}$ & 0.42 & $\begin{array}{l}\mathbf{1 . 1 1}^{*} \\
{[23.06]}\end{array}$ & 0.12 \\
\hline $\mathrm{LB}(15)$ & $\mathbf{0 . 2 9}_{[5.56]}$ & 0.20 & $\begin{array}{c}\mathbf{0 . 2 0 ^ { * }} \\
{[8.22]}\end{array}$ & 0.06 & $\begin{array}{l}0.09 \\
{[1.48]}\end{array}$ & 0.23 \\
\hline \multirow[t]{4}{*}{ Dummy } & $\begin{array}{c}-\mathbf{0 . 5 3}^{*} \\
{[-7.33]}\end{array}$ & 0.27 & $\begin{array}{c}-\mathbf{0 . 3 4} \\
{[-4.29]}\end{array}$ & 0.31 & $\begin{array}{c}-\mathbf{0 . 1 9}^{*} \\
{[-3.57]}\end{array}$ & 0.21 \\
\hline & \multicolumn{6}{|c|}{ Test Group $18(18 \times 98=1764$ observations $)$} \\
\hline & \multicolumn{2}{|c|}{ Volatility } & \multicolumn{2}{|c|}{ Noise variance } & \multicolumn{2}{|c|}{$\mathrm{SNR}_{3}$} \\
\hline & Fixed & Random & Fixed & Random & Fixed & Random \\
\hline Intercept & $\begin{array}{c}-\mathbf{7 . 0 1}^{*} \\
{[-29.43]}\end{array}$ & 1.00 & $\begin{array}{c}-\mathbf{1 4 . 7 4} \\
{[-58.50]}\end{array}$ & 1.06 & $\begin{array}{l}7.72^{*} \\
{[114.84]}\end{array}$ & 0.27 \\
\hline Nr quotes & $\begin{array}{l}\mathbf{1 . 5 0} \\
{[8.21]}\end{array}$ & 0.71 & $\begin{array}{l}0.26 \\
{[1.82]}\end{array}$ & 0.57 & $\begin{array}{l}\mathbf{1 . 1 8} \\
{[18.70]}\end{array}$ & 0.22 \\
\hline $\mathrm{LB}(15)$ & ${ }_{[3.65]}^{0.24^{*}}$ & 0.25 & $\mathbf{0 . 1 3}^{*}$ & 0.09 & $\begin{array}{l}\mathbf{0 . 1 0} \\
{[2.10]}\end{array}$ & 0.19 \\
\hline \multirow[t]{4}{*}{ Dummy } & $\begin{array}{c}-\mathbf{0 . 8 3}^{*} \\
{[-7.46]}\end{array}$ & 0.44 & $\begin{array}{c}-\mathbf{0 . 7 1} \\
{[-7.12]}\end{array}$ & 0.40 & $\begin{array}{c}-\mathbf{0 . 1 1} \\
{[-2.48]}\end{array}$ & 0.16 \\
\hline & \multicolumn{6}{|c|}{ Test Group Dow Jones $(30 \times 98=2940$ observations $)$} \\
\hline & \multicolumn{2}{|c|}{ Volatility } & \multicolumn{2}{|c|}{ Noise variance } & \multicolumn{2}{|c|}{$\mathrm{SNR}_{3}$} \\
\hline & Fixed & Random & Fixed & Random & Fixed & Random \\
\hline Intercept & $\begin{array}{c}-\mathbf{7 . 4 3}^{*} \\
{[-70.30]}\end{array}$ & 0.57 & $\begin{array}{c}\mathbf{- 1 6 . 0 2} \\
{[-149.35]}\end{array}$ & 0.58 & $\begin{array}{l}8.57^{*} \\
{[310.72]}\end{array}$ & 0.14 \\
\hline Nr quotes & $\mathbf{0 . 6 6}^{\mathbf{0}}{ }^{*}$ & 0.69 & $\begin{array}{c}-\mathbf{0 . 2 9}^{*} \\
{[-2.67]}\end{array}$ & 0.54 & $\begin{array}{c}\mathbf{0 . 8 9}^{*} \\
{[18.43]}\end{array}$ & 0.18 \\
\hline $\mathrm{LB}(15)$ & $\begin{array}{c}\mathbf{0 . 4 8} \\
{[22.53]}\end{array}$ & 0.08 & $\begin{array}{l}\mathbf{0 . 2 3 ^ { * }} \\
{[14.86]}\end{array}$ & 0.06 & $\begin{array}{c}\mathbf{0 . 2 7} \\
{[19.05]}\end{array}$ & 0.06 \\
\hline Dummy & $\begin{array}{c}-\mathbf{0 . 5 0} \\
{[-9.78]}\end{array}$ & 0.24 & $\begin{array}{c}-\mathbf{0 . 5 0} \\
{[-8.30]}\end{array}$ & 0.32 & $\begin{array}{l}0.01 \\
{[0.44]}\end{array}$ & 0.11 \\
\hline
\end{tabular}

Note: Bolded significant at $5 \%$ level, ${ }^{*}$ denotes $1 \%$ ( $t$-statistics in brackets). The random part denotes the variable's standard deviation (covariances not reported). 
Table 13: Number of jumps for CG18 (above) and TG18 (below).

\begin{tabular}{|c|c|c|c|c|c|c|c|c|c|c|c|c|}
\hline \multirow[t]{3}{*}{ Stock } & \multicolumn{6}{|c|}{ Before decimalization } & \multicolumn{6}{|c|}{ After decimalization } \\
\hline & \multicolumn{3}{|c|}{ Threshold $^{q}$} & \multicolumn{3}{|c|}{ Threshold $^{t}$} & \multicolumn{3}{|c|}{ Threshold $^{q}$} & \multicolumn{3}{|c|}{ Threshold $^{t}$} \\
\hline & .01 & .02 & .03 & .01 & .02 & .03 & .01 & .02 & .03 & .01 & .02 & .03 \\
\hline $\mathrm{APC}$ & 5 & 1 & - & 16 & 2 & - & 1 & - & - & 5 & 1 & - \\
\hline BEN & 1 & - & - & 4 & - & - & 10 & - & - & & - & - \\
\hline CI & 14 & - & - & 8 & 1 & - & 2 & - & - & 4 & - & - \\
\hline CL & - & - & - & 1 & - & - & - & - & - & 1 & - & - \\
\hline $\mathrm{CPQ}$ & 1 & - & - & 28 & 1 & - & 1 & - & - & 19 & 1 & - \\
\hline DCX & 5 & - & - & 3 & 1 & - & - & - & - & 2 & - & - \\
\hline FDX & 6 & 1 & - & 13 & 1 & 1 & 6 & 1 & - & 8 & 1 & 1 \\
\hline GMH & 13 & 1 & - & 38 & 2 & - & 3 & - & - & 13 & - & - \\
\hline GT & 21 & - & - & 37 & 1 & - & 2 & - & - & 15 & - & - \\
\hline GTW & 51 & 2 & 1 & 152 & 21 & 2 & 7 & - & - & 33 & 1 & - \\
\hline $\mathrm{H}$ & - & - & - & - & - & - & 1 & - & - & - & - & - \\
\hline IOM & 146 & 15 & 3 & 391 & 29 & 3 & 126 & 5 & - & 250 & 10 & 1 \\
\hline LE & 35 & 6 & 2 & 40 & 7 & 2 & 14 & 1 & - & 14 & 3 & - \\
\hline LMT & 2 & - & - & 12 & 1 & - & 7 & - & - & 8 & - & - \\
\hline$S$ & 2 & - & - & 7 & - & - & 5 & - & - & 1 & - & - \\
\hline SGY & 10 & 2 & - & 16 & 4 & 1 & 9 & - & - & 8 & - & - \\
\hline STT & - & - & - & 4 & - & - & 1 & - & - & 5 & - & - \\
\hline UBS & - & - & - & 1 & - & - & 9 & - & - & 1 & - & - \\
\hline Total & 312 & 28 & 6 & 771 & 71 & 9 & 204 & 7 & - & 387 & 17 & 2 \\
\hline APA & - & - & - & 3 & - & - & 3 & 1 & - & 6 & 1 & 1 \\
\hline $\mathrm{BSC}$ & 1 & - & - & 4 & - & - & 11 & 1 & - & 13 & - & - \\
\hline WLP & 2 & - & - & 3 & - & - & 2 & - & - & 5 & - & - \\
\hline AVP & 3 & - & - & 2 & - & - & 8 & - & - & 2 & 1 & - \\
\hline EMC & 12 & 1 & 1 & 46 & 4 & 1 & 55 & 4 & 1 & 190 & 24 & 3 \\
\hline HDI & 4 & - & - & 12 & 1 & - & 5 & - & - & 5 & - & - \\
\hline UPS & - & - & - & & - & - & - & - & - & 1 & - & - \\
\hline $\mathrm{F}$ & 2 & - & - & 6 & - & - & - & - & - & 2 & - & - \\
\hline CTB & 17 & - & - & 49 & 1 & - & 46 & 3 & - & 43 & 2 & - \\
\hline LXK & 24 & 2 & - & 39 & 5 & 1 & 18 & - & - & 31 & 4 & - \\
\hline $\mathrm{AM}$ & 112 & 1 & - & 312 & 9 & - & 23 & 2 & 1 & 26 & 3 & - \\
\hline DBD & 16 & - & - & 30 & - & - & 43 & 1 & - & 12 & 1 & - \\
\hline SKS & 113 & 4 & - & 192 & 23 & 2 & 36 & 1 & - & 83 & 7 & - \\
\hline UTX & - & - & - & 1 & - & - & - & - & - & 2 & - & - \\
\hline TGT & 3 & - & - & 10 & - & - & 1 & - & - & 13 & - & - \\
\hline NFX & 19 & 2 & - & 13 & 1 & - & 18 & - & - & 25 & 4 & 1 \\
\hline MEL & 1 & - & - & 3 & - & - & 6 & 1 & - & 4 & 1 & - \\
\hline SNV & 1 & - & - & 2 & - & - & 10 & 2 & - & 7 & 1 & - \\
\hline Total & 330 & 10 & 1 & 727 & 44 & 4 & 285 & 16 & 2 & 470 & 49 & 5 \\
\hline
\end{tabular}


Table 14: Number of jumps for TGDJ.

\begin{tabular}{|c|c|c|c|c|c|c|c|c|c|c|c|c|}
\hline \multirow[t]{3}{*}{ Stock } & \multicolumn{6}{|c|}{ Before decimalization } & \multicolumn{6}{|c|}{ After decimalization } \\
\hline & \multicolumn{3}{|c|}{ Threshold $^{q}$} & \multicolumn{3}{|c|}{ Threshold $^{t}$} & \multicolumn{3}{|c|}{ Threshold $^{q}$} & \multicolumn{3}{|c|}{ Threshold $^{t}$} \\
\hline & .01 & .02 & .03 & .01 & .02 & .03 & .01 & .02 & .03 & .01 & .02 & .03 \\
\hline AA & - & - & - & 2 & - & - & - & - & - & 5 & - & - \\
\hline AIG & - & - & - & 1 & - & - & - & - & - & - & - & - \\
\hline AXP & 8 & 1 & - & 13 & 2 & 1 & 8 & 1 & 1 & 14 & 2 & - \\
\hline BA & - & - & - & 1 & - & - & - & - & - & 1 & - & - \\
\hline $\mathrm{C}$ & 2 & - & - & 7 & - & - & 7 & - & - & 5 & - & - \\
\hline CAT & - & - & - & 2 & - & - & 1 & - & - & 1 & - & - \\
\hline $\mathrm{DD}$ & - & - & - & - & - & - & 2 & - & - & 1 & - & - \\
\hline DIS & 8 & 1 & - & 10 & - & - & 2 & - & - & 5 & - & - \\
\hline EK & - & - & - & 3 & - & - & 4 & - & - & 4 & 1 & - \\
\hline GE & - & - & - & 4 & - & - & 2 & - & - & - & - & - \\
\hline GM & 4 & - & - & 3 & - & - & 3 & - & - & 3 & 1 & - \\
\hline $\mathrm{HD}$ & 2 & - & - & 8 & 1 & 1 & - & - & - & 6 & - & - \\
\hline HON & 1 & - & - & - & - & - & 1 & - & - & 2 & - & - \\
\hline HWP & 7 & 1 & - & 32 & 3 & - & 5 & - & - & 10 & - & - \\
\hline IBM & 3 & 2 & - & 5 & 1 & 1 & - & - & - & 1 & - & - \\
\hline IP & 2 & - & - & 5 & - & - & 4 & 1 & - & 10 & - & - \\
\hline JNJ & - & - & - & - & - & - & - & - & - & - & - & - \\
\hline $\mathrm{KO}$ & - & - & - & - & - & - & 6 & - & - & 3 & - & - \\
\hline LU & 4 & - & - & 8 & 1 & - & 34 & 11 & 6 & 54 & 17 & 14 \\
\hline MCD & - & - & - & - & - & - & 1 & - & - & 4 & - & - \\
\hline MMM & - & - & - & - & - & - & 1 & - & - & 4 & - & - \\
\hline MO & 2 & - & - & 8 & 1 & - & - & - & - & 2 & - & - \\
\hline MRK & - & - & - & 3 & - & - & - & - & - & 3 & - & - \\
\hline PFE & - & - & - & 5 & - & - & - & - & - & 1 & - & - \\
\hline PG & - & - & - & - & - & - & 1 & - & - & 4 & 1 & - \\
\hline SBC & 1 & - & - & 3 & - & - & - & - & - & - & - & - \\
\hline $\mathrm{T}$ & 3 & - & - & 9 & - & - & - & - & - & 2 & - & - \\
\hline VZ & 1 & - & - & 2 & - & - & 1 & - & - & 7 & - & - \\
\hline WMT & 1 & - & - & 2 & 1 & - & 1 & - & - & 4 & - & - \\
\hline $\mathrm{XOM}$ & - & - & - & - & - & - & - & - & - & - & - & - \\
\hline Total & 49 & 5 & 0 & 136 & 10 & 3 & 84 & 13 & 7 & 156 & 22 & 14 \\
\hline
\end{tabular}




\section{A Appendix}

\section{A.1 Subsampling and averaging}

The TSRV estimator of Zhang, Mykland, and Aït-Sahalia (2005) is defined as

$$
\widehat{\langle X, X}_{T}^{(t s r v)}=[Y, Y]_{T}^{(K)}-\frac{\bar{n}}{n}[Y, Y]_{T}^{(a l l)}
$$

where

$$
[Y, Y]_{T}^{(K)}=\frac{1}{K} \sum_{i=0}^{n-K}\left(Y_{t_{i+K}}-Y_{t_{i}}\right)^{2},
$$

$\bar{n}=(n-K+1) / K$, and $1<K \leq n$ (see Section 2$)$.

Figure below illustrates two numerically equal ways to calculate the first sum in the TSRV with $K=5$. The axis shows the time of trade (or quote update); there are now 11 of them. In the lower panel, price is (sub)sampled 5 events apart. 5 different sums of squared returns are then formed and finally averaged to get $[Y, Y]_{T}^{(5)}$. This process is called subsampling and averaging. The upper panel illustrates a more straightforward way to calculate the same quantity by just smoothly sliding a "window" of length 5 through the trading day. There are then 6 terms to be squared, summed, and to be finally divided by 5 (to get the "average").

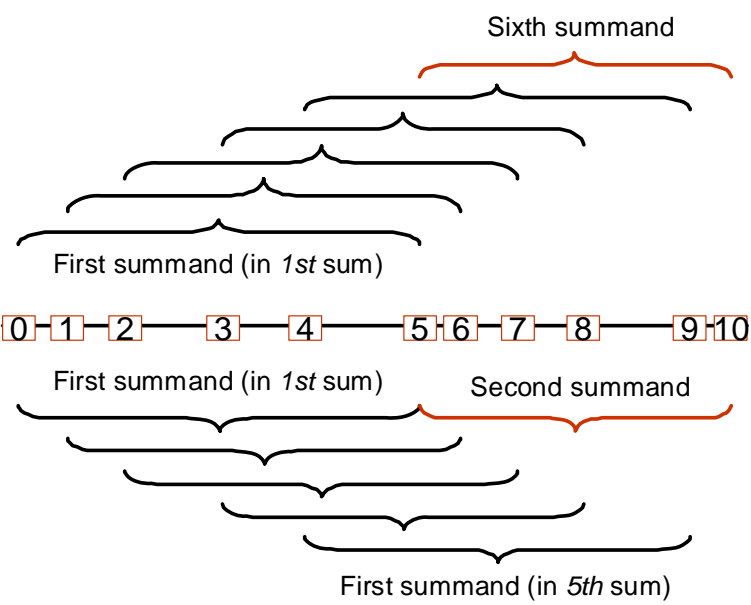

Two numerically equal ways to calculate the first sum in the TSRV $(K=5)$. 


\section{A.2 A list of the pilot decimalized stocks}

All pilot decimalized stocks and their times of decimalization.

\begin{tabular}{|c|c|c|c|c|c|c|}
\hline$\overline{\mathrm{Aug} / 28 / 00}$ & \multicolumn{2}{|c|}{$\overline{\mathrm{Sep} / 25 / 00}$} & \multicolumn{4}{|c|}{$\overline{\mathrm{Dec} / 04 / 00}$} \\
\hline APC & ACR & $\mathbf{L E}$ & $\mathrm{ABX}$ & $\overline{\mathrm{DUC}}$ & MUE & VTS \\
\hline FCEA & $\mathrm{ADO}$ & LMT & AHP & $\mathrm{EF}$ & MWY & WLK \\
\hline FCEB & AOL & LSS & ASH & ESA & MYS & WLV \\
\hline FDX & ASF & MHI & ASP & EXC & $\mathrm{N}$ & WPC \\
\hline GTW & AXM & MLM & ATW & EXEA & NPRE & WS \\
\hline HUG & BEN & MMR & AWG & FUN & NSH & WXS \\
\hline MNS & BGS & MTB & $\mathrm{BBC}$ & FVH & NVB & ZTR \\
\hline & CBU & NHL & BBCPRA & GDI & OMS & \\
\hline & CI & NSS & BLC & GLT & PEPRX & \\
\hline & CL & PTZ & BLU & GRO & POM & \\
\hline & CPQ & RCL & $\mathrm{BN}$ & GVT & POMPRT & \\
\hline & CXH & $\mathbf{S}$ & BVF & HAT & PVD & \\
\hline & DA & SGY & BVFPR & HCA & RBK & \\
\hline & DAJ & $\mathrm{SH}$ & BVFWS & HED & REV & \\
\hline & DCX & SRF & BXM & IT & $\mathrm{SE}$ & \\
\hline & DON & SRH & BYH & ITB & SFD & \\
\hline & DTF & STOPRE & CAGPRA & KMB & SIE & \\
\hline & EN & STT & CAGPRB & LHP & SJM & \\
\hline & FDS & TAI & CAGPRC & LSI & SLR & \\
\hline & GMH & $\mathrm{TM}$ & CGI & LUV & SVR & \\
\hline & GT & TRC & CLM & MAD & SWM & \\
\hline & $\mathbf{H}$ & TWX & CMM & MAT & SYK & \\
\hline & HAR & TWXPRT & CMMPRB & MCK & TBC & \\
\hline & $\mathrm{HBC}$ & TXA & CMMPRF & MDC & TDR & \\
\hline & HPRA & UBS & CMMPRG & MI & TMO & \\
\hline & HYP & VAL & CYH & MIC & TVX & \\
\hline & IOM & VIG & DLX & MPR & VFC & \\
\hline & $\mathrm{KF}$ & WSO & DOM & MQY & VGM & \\
\hline & KSM & & DRF & MTR & VLT & \\
\hline
\end{tabular}

Note: Only the bolded stocks in August and September are active enough. 
A.3 Two-scale volatility estimates 
TSRV volatility estimates for CG18 (above) and TG18 (below).

\begin{tabular}{|c|c|c|c|c|c|c|c|c|}
\hline Stock & \multicolumn{4}{|c|}{ Before decimalization } & \multicolumn{4}{|c|}{ After decimalization } \\
\hline & $\mathrm{TSRV}^{t}$ & $\left(\%^{q}\right)$ & $\left(\%_{r v}^{t}\right)$ & $\left(\%_{r v}^{q}\right)$ & $\mathrm{TSRV}^{t}$ & $\left(\%^{q}\right)$ & $\left(\%_{r v}^{t}\right)$ & $\left(\%_{r v}^{q}\right)$ \\
\hline APC & $7.92 e-04$ & 5 & 23 & 42 & $\downarrow 5.42 e-04$ & 7 & 9 & 32 \\
\hline BEN & $5.06 e-04$ & -3 & 16 & 22 & $\downarrow 4.81 e-04$ & -2 & 22 & 8 \\
\hline CI & $5.67 e-04$ & -3 & 6 & 1 & $\downarrow 4.20 e-04$ & 1 & -2 & -23 \\
\hline CL & $4.52 e-04$ & 1 & 33 & 46 & $\downarrow 3.35 e-04$ & 1 & 1 & 22 \\
\hline $\mathrm{CPQ}$ & $1.61 e-03$ & 3 & -57 & 52 & $\downarrow 1.48 e-03$ & 4 & -19 & 47 \\
\hline DCX & $3.08 e-04$ & 4 & -129 & -14 & $\downarrow 1.84 e-04$ & -1 & -65 & -23 \\
\hline FDX & $5.31 e-04$ & 8 & 8 & 35 & $\downarrow 4.88 e-04$ & 2 & -12 & 13 \\
\hline GMH & $1.43 e-03$ & 4 & -7 & 30 & $\downarrow 9.65 e-04$ & 3 & -41 & 14 \\
\hline GT & $6.32 e-04$ & -2 & -47 & -37 & $\downarrow 5.32 e-04$ & 0 & -60 & -49 \\
\hline GTW & $2.49 e-03$ & 5 & -7 & 32 & $\downarrow 2.45 e-03$ & 3 & 20 & 42 \\
\hline $\mathrm{H}$ & $1.70 e-05$ & -25 & -273 & -185 & $\downarrow 1.51 e-05$ & -15 & -89 & -85 \\
\hline IOM & $2.17 e-03$ & 2 & -122 & -22 & $\downarrow 9.56 e-04$ & -13 & -179 & -118 \\
\hline $\mathrm{LE}$ & $1.07 e-03$ & -1 & 33 & 24 & $\downarrow 7.37 e-04$ & 3 & 25 & 21 \\
\hline LMT & $7.82 e-04$ & 1 & -18 & 6 & $\downarrow 6.06 e-04$ & 3 & -30 & 2 \\
\hline S & $5.74 e-04$ & -2 & 5 & 33 & $\downarrow 4.21 e-04$ & -1 & -6 & 2 \\
\hline SGY & $5.74 e-04$ & 10 & 30 & 38 & $5.93 e-04$ & 3 & 25 & 31 \\
\hline STT & $6.97 e-04$ & 1 & 12 & 49 & $7.21 e-04$ & 7 & -9 & 26 \\
\hline UBS & $9.27 e-05$ & 7 & -6 & 7 & $1.25 e-04$ & -2 & 3 & -23 \\
\hline Avg & $8.50 e-04$ & 1 & -28 & 9 & $16.70 e-04$ & 0 & -23 & -3 \\
\hline APA & $5.87 e-04$ & 4 & -8 & 26 & $\downarrow 4.93 e-04$ & 6 & 8 & 21 \\
\hline $\mathrm{BSC}$ & $8.03 e-04$ & 6 & -47 & 9 & $8.65 e-04$ & 4 & -18 & -12 \\
\hline WLP & $8.43 e-04$ & 1 & 17 & 36 & $\downarrow 5.51 e-04$ & 5 & 21 & 29 \\
\hline AVP & $6.02 e-04$ & 0 & -26 & 21 & $\downarrow 4.06 e-04$ & -3 & 14 & 20 \\
\hline EMC & $2.30 e-03$ & 1 & -18 & 32 & $3.31 e-03$ & -5 & -16 & 10 \\
\hline HDI & $9.65 e-04$ & 3 & -16 & 37 & $\downarrow 6.90 e-04$ & 5 & -21 & 24 \\
\hline UPS & $2.14 e-04$ & 0 & -90 & 11 & $\downarrow 1.43 e-04$ & 7 & -66 & -20 \\
\hline $\mathrm{F}$ & $6.92 e-04$ & 4 & -224 & 9 & $\downarrow 4.49 e-04$ & 7 & -66 & 18 \\
\hline CTB & $4.45 e-04$ & -16 & -410 & -110 & $6.90 e-04$ & -13 & -60 & -83 \\
\hline LXK & $1.75 e-03$ & 1 & 13 & 39 & $\downarrow 1.21 e-03$ & 2 & 27 & 29 \\
\hline $\mathrm{AM}$ & $1.22 e-03$ & -9 & -239 & -46 & $\downarrow 7.00 e-04$ & -7 & -4 & -7 \\
\hline DBD & $8.86 e-04$ & -4 & -13 & 3 & $\downarrow 4.40 e-04$ & -18 & 13 & -84 \\
\hline SKS & $9.46 e-04$ & -5 & -218 & -78 & $\downarrow 6.95 e-04$ & 3 & -73 & -24 \\
\hline UTX & $4.78 e-04$ & 2 & -4 & 37 & $\downarrow 4.52 e-04$ & -1 & 23 & 36 \\
\hline TGT & $9.35 e-04$ & 3 & -92 & 18 & $\downarrow 8.59 e-04$ & 7 & -5 & 34 \\
\hline NFX & $6.70 e-04$ & 6 & -6 & 7 & $\downarrow 6.50 e-04$ & 3 & -1 & 15 \\
\hline MEL & $5.76 e-04$ & 2 & -79 & 23 & $\downarrow 5.28 e-04$ & 5 & -8 & 31 \\
\hline SNV & $2.67 e-04$ & 0 & -219 & -53 & $3.04 e-04$ & 2 & -30 & -23 \\
\hline Avg & $8.44 e-04$ & $\overline{0}$ & -93 & 1 & $\downarrow 7.47 e-04$ & 0 & -15 & 1 \\
\hline
\end{tabular}

Note: Bolded percentages denote estimates that have become closer to $\mathrm{TSRV}^{t}$ : $\%^{q}=\frac{T S R V^{t}-T S R V^{q}}{T S R V^{t}} \times 100 ; \%_{r v}^{t}=\frac{T S R V^{t}-R V^{t}}{T S R V^{t}} \times 100 ; \%_{r v}^{q}=\frac{T S R V^{t}-R V^{q}}{T S R V^{t}} \times 100$. 
TSRV volatility estimates for TGDJ.

\begin{tabular}{|c|c|c|c|c|c|c|c|c|}
\hline "Stock & \multicolumn{4}{|c|}{$\overline{\text { Before decimalization }}$} & \multicolumn{4}{|c|}{ After decimalization } \\
\hline & $\mathrm{TSRV}^{t}$ & $\left(\%^{q}\right)$ & $\left(\%_{r v}^{t}\right)$ & $\left(\%_{r v}^{q}\right)$ & $\mathrm{TSRV}^{t}$ & $\left(\%^{q}\right)$ & $\left(\%_{r v}^{t}\right)$ & $\left(\%_{v v}^{q}\right)$ \\
\hline AA & $8.62 e-04$ & 2 & -137 & 12 & $\downarrow 5.43 e-04$ & 3 & -37 & 30 \\
\hline AIG & $3.97 e-04$ & 2 & -31 & 39 & $\downarrow 3.03 e-04$ & 5 & -14 & 24 \\
\hline AXP & $9.17 e-04$ & 2 & -68 & 26 & $\downarrow 8.79 e-04$ & 9 & 1 & 34 \\
\hline BA & $6.12 e-04$ & 3 & -5 & 49 & $\downarrow 4.83 e-04$ & 2 & 6 & 37 \\
\hline $\mathrm{C}$ & $8.38 e-04$ & 4 & -123 & 35 & $\downarrow 7.67 e-04$ & 7 & -28 & 4 \\
\hline CAT & $4.97 e-04$ & -1 & -79 & 16 & $\downarrow 4.33 e-04$ & 3 & 2 & 21 \\
\hline DD & $6.16 e-04$ & 3 & -173 & 14 & $\downarrow 4.46 e-04$ & 5 & -25 & 36 \\
\hline DIS & $6.85 e-04$ & 3 & -272 & 2 & $\downarrow 6.65 e-04$ & 7 & -44 & 29 \\
\hline EK & $6.26 e-04$ & -2 & -56 & 26 & $\downarrow 5.34 e-04$ & 2 & 18 & 27 \\
\hline GE & $5.07 e-04$ & 2 & -287 & 20 & $5.11 e-04$ & 3 & -37 & 35 \\
\hline GM & $5.75 e-04$ & 3 & -29 & 34 & $\downarrow 3.69 e-04$ & 2 & 9 & 45 \\
\hline $\mathrm{HD}$ & $8.22 e-04$ & 5 & -203 & 30 & $\downarrow 7.55 e-04$ & 2 & -25 & 45 \\
\hline $\mathrm{HON}$ & $5.06 e-04$ & 2 & -78 & 2 & $\downarrow 5.73 e-04$ & 4 & -19 & 2 \\
\hline HWP & $1.76 e-03$ & 2 & -135 & 16 & $\downarrow 1.33 e-03$ & 2 & -65 & 21 \\
\hline IBM & $8.74 e-04$ & 3 & -59 & 33 & $\downarrow 6.99 e-04$ & 2 & -17 & 32 \\
\hline IP & $7.89 e-04$ & 1 & -80 & 17 & $\downarrow 6.98 e-04$ & 5 & -29 & 23 \\
\hline JNJ & $2.58 e-04$ & 4 & -95 & 32 & $\downarrow 2.02 e-04$ & 5 & -26 & 39 \\
\hline $\mathrm{KO}$ & $3.63 e-04$ & 3 & -114 & 27 & $4.55 e-04$ & 2 & 13 & 38 \\
\hline $\mathrm{LU}$ & $2.07 e-03$ & 5 & -941 & 7 & $2.52 e-03$ & -8 & -95 & 18 \\
\hline MCD & $4.26 e-04$ & 3 & -336 & 0 & $4.26 e-04$ & 6 & -88 & 23 \\
\hline MMM & $4.12 e-04$ & 3 & 13 & 45 & $4.90 e-04$ & 5 & 22 & 39 \\
\hline MO & $6.57 e-04$ & 2 & -119 & 35 & $\downarrow 3.75 e-04$ & 4 & 9 & 52 \\
\hline MRK & $3.64 e-04$ & 2 & -68 & 33 & $\downarrow 3.55 e-04$ & 3 & 4 & 30 \\
\hline PFE & $5.29 e-04$ & 5 & -456 & -11 & $\downarrow 3.95 e-04$ & 5 & -126 & 18 \\
\hline PG & $4.67 e-04$ & 6 & -69 & 25 & $\downarrow 4.03 e-04$ & 6 & -45 & 19 \\
\hline SBC & $5.02 e-04$ & 2 & -112 & 33 & $6.55 e-04$ & 3 & 23 & 62 \\
\hline $\mathrm{T}$ & $7.73 e-04$ & 3 & -707 & -5 & $\downarrow 6.72 e-04$ & 4 & -29 & 49 \\
\hline VZ & $4.81 e-04$ & 2 & -145 & 5 & $6.24 e-04$ & 5 & -26 & 16 \\
\hline WMT & $7.17 e-04$ & 3 & -123 & 28 & $\downarrow 6.61 e-04$ & 3 & 6 & 43 \\
\hline XOM & $2.48 e-04$ & 3 & -70 & 33 & $2.15 e-04$ & 7 & 3 & 38 \\
\hline Avg & $6.71 e-04$ & 3 & -172 & 22 & $6.14 e-04$ & 4 & -22 & 31 \\
\hline
\end{tabular}

Note: Bolded percentages denote estimates that have become closer to TSRV ${ }^{t}$ : $\%^{q}=\frac{T S R V^{t}-T S R V^{q}}{T S R V^{t}} \times 100 ; \%_{r v}^{t}=\frac{T S R V^{t}-R V^{t}}{T S R V^{t}} \times 100 ; \%_{r v}^{q}=\frac{T S R V^{t}-R V^{q}}{T S R V^{t}} \times 100$. 
GTSRV volatility estimates for CG18 (above) and TG18 (below).

\begin{tabular}{|c|c|c|c|c|c|c|c|c|}
\hline "Stock & \multicolumn{4}{|c|}{ "Before decimalization } & \multicolumn{4}{|c|}{ After decimalization } \\
\hline & GTSRV $^{t}$ & $\left(\%^{q}\right)$ & $\left(\%_{r v}^{t}\right)$ & $\left(\%_{r v}^{q}\right)$ & GTSRV $^{t}$ & $\left(\%^{q}\right)$ & $\left(\%_{r v}^{t}\right)$ & $\left(\%_{r v}^{q}\right)$ \\
\hline $\mathrm{APC}$ & $7.80 e-04$ & 1 & 21 & 41 & $\downarrow 5.37 e-04$ & 5 & 8 & 32 \\
\hline $\mathrm{BEN}$ & $4.92 e-04$ & -9 & 14 & 20 & $\downarrow 4.71 e-04$ & -7 & 20 & 6 \\
\hline $\mathrm{CI}$ & $5.57 e-04$ & -7 & 4 & -1 & $\downarrow 4.13 e-04$ & -3 & -4 & -25 \\
\hline $\mathrm{CL}$ & $4.43 e-04$ & -2 & 32 & 45 & $\downarrow 3.30 e-04$ & -2 & -1 & 20 \\
\hline $\mathrm{CPQ}$ & $1.59 e-03$ & 0 & -59 & 51 & $\downarrow 1.46 e-03$ & 1 & -21 & 46 \\
\hline DCX & $2.85 e-04$ & -10 & -148 & -23 & $\downarrow 1.74 e-04$ & -12 & -75 & -31 \\
\hline FDX & $5.23 e-04$ & 5 & 7 & 34 & $\downarrow 4.78 e-04$ & -3 & -15 & 12 \\
\hline GMH & $1.40 e-03$ & -1 & -10 & 28 & $\downarrow 9.37 e-04$ & -3 & -45 & 11 \\
\hline GT & $6.05 e-04$ & -12 & -54 & -44 & $\downarrow 5.09 e-04$ & -8 & -67 & -55 \\
\hline GTW & $2.44 e-03$ & 1 & -9 & 31 & $\downarrow 2.41 e-03$ & 0 & 18 & 41 \\
\hline $\mathrm{H}$ & $1.54 e-05$ & -66 & -312 & -215 & $\downarrow 1.43 e-05$ & -33 & -100 & -96 \\
\hline IOM & $1.99 e-03$ & -17 & -142 & -32 & $\downarrow 9.19 e-04$ & -36 & -191 & -127 \\
\hline LE & $1.02 e-03$ & -13 & 30 & 20 & $\downarrow 7.24 e-04$ & -4 & 23 & 19 \\
\hline LMT & $7.62 e-04$ & -5 & -22 & 3 & $\downarrow 5.92 e-04$ & -2 & -33 & 0 \\
\hline $\mathrm{S}$ & $5.60 e-04$ & -6 & 3 & 31 & $\downarrow 4.12 e-04$ & -6 & -8 & 0 \\
\hline SGY & $5.56 e-04$ & 2 & 28 & 36 & $5.68 e-04$ & -6 & 22 & 28 \\
\hline $\mathrm{STT}$ & $6.85 e-04$ & -2 & 11 & 48 & $7.08 e-04$ & 4 & -11 & 24 \\
\hline UBS & $9.00 e-05$ & -2 & -9 & 4 & $1.21 e-04$ & -12 & 1 & -26 \\
\hline Avg & $8.21 e-04$ & -8 & -34 & 4 & $\downarrow 6.54 e-04$ & -7 & -27 & -7 \\
\hline $\mathrm{APA}$ & $5.70 e-04$ & -1 & -11 & 23 & $4.85 e-04$ & 2 & 7 & 20 \\
\hline $\mathrm{BSC}$ & $7.68 e-04$ & -1 & -54 & 5 & $8.54 e-04$ & 0 & -19 & -14 \\
\hline WLP & $8.31 e-04$ & -3 & 16 & 35 & $\downarrow 5.41 e-04$ & 1 & 20 & 28 \\
\hline AVP & $5.80 e-04$ & -7 & -31 & 18 & $\downarrow 3.96 e-04$ & -7 & 12 & 18 \\
\hline EMC & $2.28 e-03$ & 0 & -19 & 32 & $3.28 e-03$ & -7 & -18 & 9 \\
\hline HDI & $9.34 e-04$ & -2 & -20 & 35 & $\downarrow 6.76 e-04$ & 1 & -23 & 22 \\
\hline UPS & $2.00 e-04$ & -11 & -103 & 6 & $\downarrow 1.36 e-04$ & -2 & -75 & -26 \\
\hline $\mathrm{F}$ & $6.65 e-04$ & -2 & -238 & 5 & $\downarrow 4.37 e-04$ & 2 & -71 & 16 \\
\hline CTB & $3.95 e-04$ & -49 & -475 & -136 & $6.57 e-04$ & -27 & -68 & -92 \\
\hline LXK & $1.72 e-03$ & -3 & 12 & 38 & $\downarrow 1.19 e-03$ & -2 & 25 & 27 \\
\hline $\mathrm{AM}$ & $1.10 e-03$ & -33 & -277 & -62 & $\downarrow 6.73 e-04$ & -18 & -9 & -11 \\
\hline DBD & $8.58 e-04$ & -15 & -16 & 0 & $\downarrow 4.31 e-04$ & -32 & 11 & -88 \\
\hline SKS & $8.84 e-04$ & -30 & -240 & -91 & $\downarrow 6.59 e-04$ & -11 & -82 & -31 \\
\hline UTX & $4.69 e-04$ & -2 & -6 & 35 & $\downarrow 4.44 e-04$ & -4 & 22 & 35 \\
\hline TGT & $9.06 e-04$ & -3 & -99 & 15 & $\downarrow 8.45 e-04$ & 4 & -6 & 33 \\
\hline NFX & $6.51 e-04$ & -4 & -9 & 5 & $\downarrow 6.42 e-04$ & -3 & -2 & 13 \\
\hline MEL & $5.54 e-04$ & -4 & -86 & 20 & $\downarrow 5.18 e-04$ & 2 & -9 & 30 \\
\hline SNV & $2.38 e-04$ & -21 & -258 & -71 & $2.97 e-04$ & -4 & -34 & -26 \\
\hline Avg & $8.12 e-04$ & -11 & -106 & -5 & $\downarrow 7.31 e-04$ & -6 & -18 & -2 \\
\hline
\end{tabular}

Note: Bolded percentages denote estimates that have become closer to GTSRV ${ }^{t}$ : $\%^{q}=\frac{G T S R V^{t}-G T S R V^{q}}{G T S R V^{t}} \times 100 ; \%_{r v}^{t}=\frac{G T S R V^{t}-R V^{t}}{G T S R V^{t}} \times 100 ; \%_{r v}^{q}=\frac{G T S R V^{t}-R V^{q}}{G T S R V^{t}} \times 100$. 
GTSRV volatility estimates for TGDJ.

\begin{tabular}{|c|c|c|c|c|c|c|c|c|}
\hline Stock & $\overline{\text { Befor }}$ & decin & alizatio & & After & decims & $\overline{\text { lization }}$ & \\
\hline & GTSRV $^{t}$ & $\left(\%^{q}\right)$ & $\left(\%_{r v}^{t}\right)$ & $\left(\%_{r v}^{q}\right)$ & GTSRV $^{t}$ & $\left(\%{ }^{q}\right)$ & $\left(\%_{r v}^{t}\right)$ & $\left(\%_{r v}^{q}\right)$ \\
\hline AA & $8.35 e-04$ & -4 & -144 & 9 & $5.31 e-04$ & 0 & -40 & 29 \\
\hline $\mathrm{AIG}$ & $3.90 e-04$ & -1 & -33 & 38 & $\downarrow 2.99 e-04$ & 3 & -15 & 24 \\
\hline AXP & $9.00 e-04$ & -1 & -71 & 24 & $\downarrow 8.69 e-04$ & 7 & 0 & 33 \\
\hline $\mathrm{BA}$ & $6.01 e-04$ & 0 & -7 & 48 & $\downarrow 4.75 e-04$ & -1 & 4 & 36 \\
\hline $\mathrm{C}$ & $8.30 e-04$ & 3 & -125 & 35 & $\downarrow 7.55 e-04$ & 5 & -30 & 2 \\
\hline CAT & $4.76 e-04$ & -8 & -86 & 12 & $\downarrow 4.26 e-04$ & 0 & 0 & 19 \\
\hline DD & $5.98 e-04$ & -2 & -181 & 11 & $\downarrow 4.38 e-04$ & 3 & -27 & 35 \\
\hline DIS & $6.65 e-04$ & -2 & -283 & -1 & $\downarrow 6.48 e-04$ & 3 & -47 & 27 \\
\hline EK & $6.06 e-04$ & -7 & -61 & 24 & $\downarrow 5.24 e-04$ & -2 & 17 & 25 \\
\hline GE & $4.99 e-04$ & -1 & -292 & 18 & $5.04 e-04$ & 1 & -39 & 34 \\
\hline GM & $5.59 e-04$ & -1 & -32 & 32 & $\downarrow 3.63 e-04$ & 0 & 7 & 44 \\
\hline HD & $8.10 e-04$ & 2 & -207 & 29 & $\downarrow 7.45 e-04$ & 0 & -26 & 44 \\
\hline $\mathrm{HON}$ & $4.90 e-04$ & -3 & -84 & -1 & $5.62 e-04$ & 0 & -21 & 0 \\
\hline HWP & $1.72 e-03$ & -1 & -140 & 14 & $\downarrow 1.30 e-03$ & -1 & -69 & 20 \\
\hline IBM & $8.64 e-04$ & 1 & -61 & 32 & $\downarrow 6.92 e-04$ & 0 & -18 & 31 \\
\hline IP & $7.66 e-04$ & -4 & -85 & 15 & $\downarrow 6.82 e-04$ & 1 & -32 & 21 \\
\hline JNJ & $2.53 e-04$ & 1 & -100 & 31 & $\downarrow 1.99 e-04$ & 2 & -28 & 38 \\
\hline $\mathrm{KO}$ & $3.54 e-04$ & -2 & -119 & 25 & $4.49 e-04$ & 0 & 12 & 37 \\
\hline $\mathrm{LU}$ & $2.06 e-03$ & 4 & -945 & 7 & $2.49 e-03$ & -11 & -98 & 16 \\
\hline MCD & $4.10 e-04$ & -3 & -353 & -4 & $4.15 e-04$ & 2 & -93 & 21 \\
\hline MMM & $4.04 e-04$ & 0 & 11 & 44 & $4.82 e-04$ & 3 & 20 & 38 \\
\hline $\mathrm{MO}$ & $6.45 e-04$ & -1 & -123 & 34 & $\downarrow 3.70 e-04$ & 2 & 8 & 51 \\
\hline MRK & $3.59 e-04$ & -1 & -70 & 32 & $\downarrow 3.49 e-04$ & 0 & 2 & 29 \\
\hline $\mathrm{PFE}$ & $5.22 e-04$ & 2 & -462 & -12 & $\downarrow 3.88 e-04$ & 2 & -130 & 17 \\
\hline PG & $4.56 e-04$ & 2 & -73 & 23 & $\downarrow 3.94 e-04$ & 3 & -48 & 17 \\
\hline $\mathrm{SBC}$ & $4.94 e-04$ & -1 & -115 & 32 & $\downarrow 6.47 e-04$ & 1 & 23 & 61 \\
\hline $\mathrm{T}$ & $7.64 e-04$ & 0 & -717 & -6 & $\downarrow 6.59 e-04$ & 1 & -31 & 48 \\
\hline VZ & $4.70 e-04$ & -2 & -151 & 3 & $6.14 e-04$ & 3 & -28 & 14 \\
\hline WMT & $7.06 e-04$ & 1 & -127 & 27 & $\downarrow 6.51 e-04$ & 1 & 5 & 42 \\
\hline $\mathrm{XOM}$ & $2.44 e-04$ & 0 & -74 & 32 & $\downarrow 2.12 e-04$ & 5 & 1 & 37 \\
\hline Avg & $6.58 e-04$ & -1 & -177 & 20 & $\downarrow 6.04 e-04$ & 1 & -24 & 30 \\
\hline
\end{tabular}

